Flores, Hauke, van Franeker, Jan Andries, Cisewski, Boris, Leach, Harry, Van De Putte, Anton, Meesters, Erik, Bathmann, Ulrich U.V., \& Wolff, Wim

Macrofauna under sea ice and in the open surface layer of the Lazarev Sea, Southern Ocean

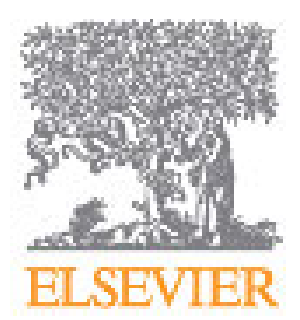

Article. Version publiée - Published version.

(C) ELSEVIER

\title{
Citation APA:
}

Flores, H., van Franeker, J. A., Cisewski, B., Leach, H., Van De Putte, A., Meesters, E., Bathmann, U. U., \& Wolff, W. (2011).

Macrofauna under sea ice and in the open surface layer of the Lazarev Sea, Southern Ocean. Deep Sea Research Part II:

Topical Studies in Oceanography, 58(19-20), 1948-1961. doi:10.1016/j.dsr2.2011.01.010

DOI: 10.1016/j.dsr2.2011.01.010

Also available at: http://hdl.handle.net/2013/ULB-DIPOT:oai:dipot.ulb.ac.be:2013/337836

Cet article publié par ELSEVIER provient du Dépôt institutionnel de l’Université Libre de Bruxelles, DIfusion http://difusion.ulb.ac.be. Il n'est accessible qu'aux membres de la communauté universitaire de l'ULB sur le réseau sécurisé de l'ULB.

Tout utilisateur autorisé peut lire, télécharger ou reproduire cet article à des fins d'usage privé ou à des fins non commerciales d'enseignement ou de recherche scientifique. Il ne peut être atteint à l'intégrité de l'article, et les noms des auteurs et de l'éditeur doivent être conservés. Tout téléchargement systématique des articles publiés par ELSEVIER mis à disposition dans DI-fusion est interdit.

This article published by ELSEVIER comes from the Institutional repository of Universite Libre de Bruxelles, DI-fusion http://difusion.ulb.ac.be. It is accessible only to the members of the university community of ULB on the ULB secure network.

Any authorized user may read, download or reproduce this article for private usage, or for non commercial research or educational purposes. The integrity of the article and identification of the author and copyright owner must be preserved. Systematic downloading of articles published by ELSEVIER that are available in DI-fusion is not permitted. 


\title{
Macrofauna under sea ice and in the open surface layer of the Lazarev Sea, Southern Ocean
}

\author{
Hauke Flores ${ }^{\mathrm{a}, *}$, Jan-Andries van Franeker ${ }^{\mathrm{a}}$, Boris Cisewski ${ }^{\mathrm{b}}$, Harry Leach ${ }^{\mathrm{c}}$, Anton P. Van de Putte $^{\mathrm{d}}$, \\ Erik (H.W.G.) Meesters ${ }^{\mathrm{a}}$, Ulrich Bathmann ${ }^{\mathrm{b}}$, Wim J. Wolff ${ }^{\mathrm{e}}$ \\ ${ }^{a}$ Institute for Marine Resources and Ecosystem Studies (IMARES), P.O. Box 167, 1790 AD Den Burg (Texel), The Netherlands \\ ${ }^{\mathrm{b}}$ Alfred Wegener Institute for Polar and Marine Research (AWI), Am Handelshafen 12, 27570 Bremerhaven, Germany \\ ${ }^{\mathrm{c}}$ Department of Earth and Ocean Sciences, University of Liverpool, 4 Brownlow Street, Liverpool L69 3 GP, United Kingdom \\ ${ }^{\mathrm{d}}$ Laboratory of Aquatic Ecology, Catholic University Leuven, Ch. Deberiotstraat 32, 3000 Leuven, Belgium

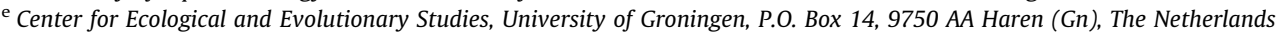

\section{A R T I C L E I N F O}

\section{Article history:}

Received 21 January 2011

Accepted 21 January 2011

Available online 2 March 2011

\section{Keywords:}

Zooplankton

Sea ice

Antarctic zone

Sympagic fauna

Community composition

Food webs

Antarctic krill

$60^{\circ} 6^{\circ} \mathrm{W} / 70^{\circ} \mathrm{S} 3^{\circ} \mathrm{E}$

\begin{abstract}
A B S T R A C T
A new fishing gear was used to sample the macrozooplankton and micronekton community in the surface layer (0-2 m) under ice and in open water, the Surface and Under Ice Trawl (SUIT). In total, 57 quantitative hauls were conducted in the Lazarev Sea (Southern Ocean) during 3 different seasons (autumn 2004, winter 2006, summer 2007/2008). At least 46 species from eight phyla were caught in all 3 seasons combined. Biomass density was dominated by Antarctic krill Euphausia superba. The average biomass density was highest under the winter sea ice and lowest under the young ice in autumn. In summer, macrozooplankton biomass was dominated by ctenophores in open water and by Antarctic krill under ice. The community composition varied significantly among seasons, and according to the presence of sea ice. The response of the community composition to the presence of sea ice was influenced by species that were significantly more abundant in open water than under ice (Cyllopus lucasii, Hyperiella dilatata), only seasonally abundant under ice (Clione antarctica), or significantly associated with sea ice (Eusirus laticarpus). A number of abundant species showed distinct diel patterns in the surface occurrence both under ice and in open water, indicating that the surface layer serves as a foraging ground predominantly at night. Our results emphasize the potential of a number of non-euphausiid macrozooplankton and micronekton species to act as energy transmitters between the production of sea ice biota and the pelagic food web. By providing a regional-scale quantitative record of macrofauna under Antarctic sea ice covering 3 seasons, this study adds new and direct evidence that the ice-water interface layer is a major functional node in the ecosystem of the Antarctic seasonal sea ice zone.
\end{abstract}

(c) 2011 Elsevier Ltd. All rights reserved.

\section{Introduction}

Large parts of the rich top predator community typical for the Southern Ocean directly or indirectly depend on resources from the surface layer (Ainley et al., 1991). For example, surface swarms of Antarctic krill Euphausia superba have been reported since the early days of Antarctic research, and whales have frequently been observed feeding at the surface (Hamner, 1982; Hardy and Gunther, 1935; Nemoto, 1983). The high biological potential of the surface layer is prominent in the Antarctic marginal sea ice zone in spring and summer, when the decay of the ice creates a shallow mixed layer, significantly enhancing primary production (Arrigo et al., 1997; Eicken, 1992). This primary production supports a diverse zooplankton community,

\footnotetext{
* Corresponding author. Tel.: +31 317487 414; fax. +31 317487362.

E-mail address: hauke.flores@wur.nl (H. Flores).
}

including Antarctic krill, salps, copepods, amphipods and a range of jelly fish and comb jelly fish, which in turn nourish fish, squid and the warm-blooded top predators (Froneman et al., 2000; Hopkins et al., 1993; Ross et al., 1998).

At least during parts of the year, the surface carries an additional substrate in the form of sea ice. Besides providing a haul-out and nursery platform for the penguins and seals of the seasonal sea-ice zone, the ice forms a habitat for a variety of sympagic ("ice-associated") lifeforms. The sympagic meiofauna community existing in the seasonally vanishing sea ice illustrates the potential of this environment as a diverse and ecologically relevant habitat (Kiko et al., 2008b; Schnack-Schiel, 2003; Schnack-Schiel et al., 2008). A rich sympagic macrofauna community exists under Arctic multi-year sea ice. Sympagic macrofauna associated with Antarctic sea ice has mainly been reported from shelf waters and fast ice (Arndt and Swadling, 2006; Gulliksen and Lonne, 1991). Recent reports of new ice-associated species in Arctic fast ice, Antarctic sea ice and at Antarctic shelf ice 
suggest that ice habitats may be more diverse than assumed to date (Bluhm et al., 2007; Kiko et al., 2008a; Watanabe et al., 2006).

Sea-ice primary and secondary production are assumed to play a key role in the seasonal sea-ice zone. Most of the primary production occurs not in the water column but in the pack-ice during large parts of the year (Arrigo and Thomas, 2004; Lizotte, 2001; McMinn et al., 2010). The productivity of the sea ice system has been proposed as playing a key role in supporting the typically high wildlife stocks of the Antarctic pack-ice zone (Ainley et al., 1986; van Franeker et al., 1997). It has been shown that copepods, larval and postlarval krill aggregate under sea ice and feed on ice algae and sympagic meiofauna (Daly, 2004; Marschall, 1988; Tanimura et al., 2008). The extent to which non-euphausiid macrofauna use the ice-water interface, however, has largely been unknown to date. If the food web of the Southern Ocean seasonal sea ice zone depends in large parts on the production of ice algae, a quantitative description of the species community in the ice-water interface layer is an important prerequisite for unraveling the trophic pathways connecting the sea ice and the pelagic systems that ultimately lead to the warmblooded top predators.

Direct quantitative evidence of macrofauna species closely associated with seasonal sea ice in Antarctic offshore waters is largely missing to date due to the difficulties imposed by the ice on conventional sampling techniques. The need to obtain a better assessment of the temporal and spatial distribution of Antarctic krill and other macrofauna has long been recognized (Siegel et al., 1990; Stretch et al., 1988). In the past decade, autonomous underwater vehicles (AUV) provided the first large-scale data on the distribution of krill under ice (Brierley et al., 2002). However, the echosounding technology used could not resolve the upper few meters of the water column, where the interaction of the pelagic and the sympagic communities is mainly expected. Although it is theoretically possible, in practice this technology is not capable of differentiating species and recording their size compositions with sufficient reliability to date.

To assess the importance of the Antarctic open surface and icewater interface layer for macrozooplankton and micronekton, a new quantitative sampling device was developed, the Surface and Under Ice Trawl (SUIT). Three expeditions in the Lazarev Sea provided the opportunity to investigate the macrofauna species composition in the surface layer of the seasonal sea ice zone both under ice and in open water in autumn, winter and summer. This study aims to

1. describe and analyze the macrozooplankton and micronekton community structure in the open surface layer and under ice during three seasons;

2. estimate the potential response of the macrozooplankton/ micronekton community to the presence of sea ice;

3. identify species that are potentially important for the trophic interaction of the sea ice with the pelagic food web;

4. discuss the ecological relevance of the ice-water interface layer and its importance for biodiversity.

\section{Material and methods}

\subsection{Data collection}

Data were collected during three research cruises of RV "Polarstern" in the Lazarev Sea in austral autumn 2004 (ANT XXI-4, $27^{\text {th }}$ March to $6^{\text {th }}$ May 2004), winter 2006 (ANT XXIII-6, $17^{\text {th }}$ June to $21^{\text {st }}$ August 2006) and summer 2007/2008 (ANT
XXIV-2, 28 ${ }^{\text {th }}$ November 2007 to $4^{\text {th }}$ February 2008). The expeditions were part of a multi-year field experiment embedded in the largely German funded LAzarev Sea KRIll Study (LAKRIS) dedicated to the investigation of the distribution, population dynamics and physiology of Antarctic krill Euphausia superba. The LAKRIS surveys sampled a regular station grid with 3-4 meridional transects with a spacing of 2-3 degrees longitude and a latitudinal station spacing of $20-30 \mathrm{~nm}$, ranging from $6^{\circ} \mathrm{W}$ to $3^{\circ} \mathrm{E}$ and from $60^{\circ} \mathrm{S}$ to the continental coast at approximately $71^{\circ} \mathrm{S}$ (Fig. 1).

Surface and Under Ice Trawls (SUIT) were used to sample macrozooplankton and micronekton in the upper $2 \mathrm{~m}$ of the water column. The net systems consisted of a steel frame with an approximately $2 \times 2 \mathrm{~m}$ net opening, with a $15 \mathrm{~m}$ long, $7 \mathrm{~mm}$ half-mesh commercial shrimp net attached to it. Floats at the top the frame kept the net at the surface or the underside of sea ice. The sampling depth was thus limited to $2 \mathrm{~m}$ in open water, or between approximately $2 \mathrm{~m}$ under new ice, and up to $5 \mathrm{~m}$ under multi-year ice. To enable sampling under undisturbed ice, an asymmetric bridle forces the net to tow off at an angle of approximately $60^{\circ}$ to starboard of the ship's track, at a cable length of $120 \mathrm{~m}$. In summer 2004, a circular plankton net (diameter $50 \mathrm{~cm}, 0.3 \mathrm{~mm}$ mesh) was mounted inside the shrimp net to sample mesozooplankton. In winter 2006 and summer $2007 / 2008$, the rear three meters of the net were lined with $0.3 \mathrm{~mm}$ plankton gauze. A strobe light and a video camera were attached to the frame. Technical details of the SUIT systems used were provided in Table 1.

An acoustic Doppler Current Profiler (ADCP) was used in winter 2006 and summer 2007/2008 to estimate the amount of water entering the net and to analyze its flow properties. ADCP current speed measurements were used to estimate the effective water distance passed $(\mathrm{eD})$ as the sum of the products of the duration [s] and the current speed $\left[\mathrm{m} \mathrm{s}^{-1}\right]$ of each measuring interval (5-20 s). For SUIT hauls conducted in autumn 2004, eD was estimated a posteriori by means of a linear regression of ADCP-estimated eD versus the ship's track distance during trawling from the 2006 and 2007/2008 current speed measurements $\left(\mathrm{eD}=0.8 \times\right.$ Ship track distance $\left.+148 \mathrm{~m} ; \mathrm{R}^{2}=0.88 ; \mathrm{p}<0.05\right)$. The surface area sampled $\left[\mathrm{m}^{2}\right]$ was calculated for each haul by multiplying eD with the opening width. A detailed description of the SUIT and its fishing properties was provided by van Franeker et al. (2009). Standard hauls lasted 25 minutes, with a minimum of 15 and a maximum of 49 minutes and an average towing speed of $1.5-2.5 \mathrm{kn}\left(0.8-1.3 \mathrm{~m} \mathrm{~s}^{-1}\right)$. During each trawl, changes in ship speed, ice coverage [\%], ice thickness [cm] and irregularities were recorded by an observer watching the net from the ship.

Of the total 75 SUIT hauls, 24 hauls were conducted in autumn 2004, 30 hauls in winter 2006 and 21 hauls in summer 2007/ 2008. For the purpose of this study data were restricted to the 71 hauls conducted on the LAKRIS grid (i.e. south of $59^{\circ} \mathrm{S}$ ). Fourteen of them were excluded from quantitative analysis because of irregularities during the tow. Forty-six of the remaining 57 quantitative hauls were conducted at night. Six hauls at three locations were dedicated to day/night comparisons in winter 2006 and in summer 2007/2008, respectively (Fig. 1).

The catch was immediately sorted on board. All macrofauna $>0.5 \mathrm{~cm}$ was collected from either the entire sample or a representative subsample. Macrozooplankton and micronekton were separated by species. Displacement volume and number of individuals of each species were noted. In each species, the number of animals caught was expressed as the density of individuals per $1,000 \mathrm{~m}^{2}$ surface area $\left[\mathrm{N} 1,000 \mathrm{~m}^{-2}\right.$ ]. Euphausiid furcilia larvae and copepods were excluded from the present analysis, because we have not yet assessed the quantitative 


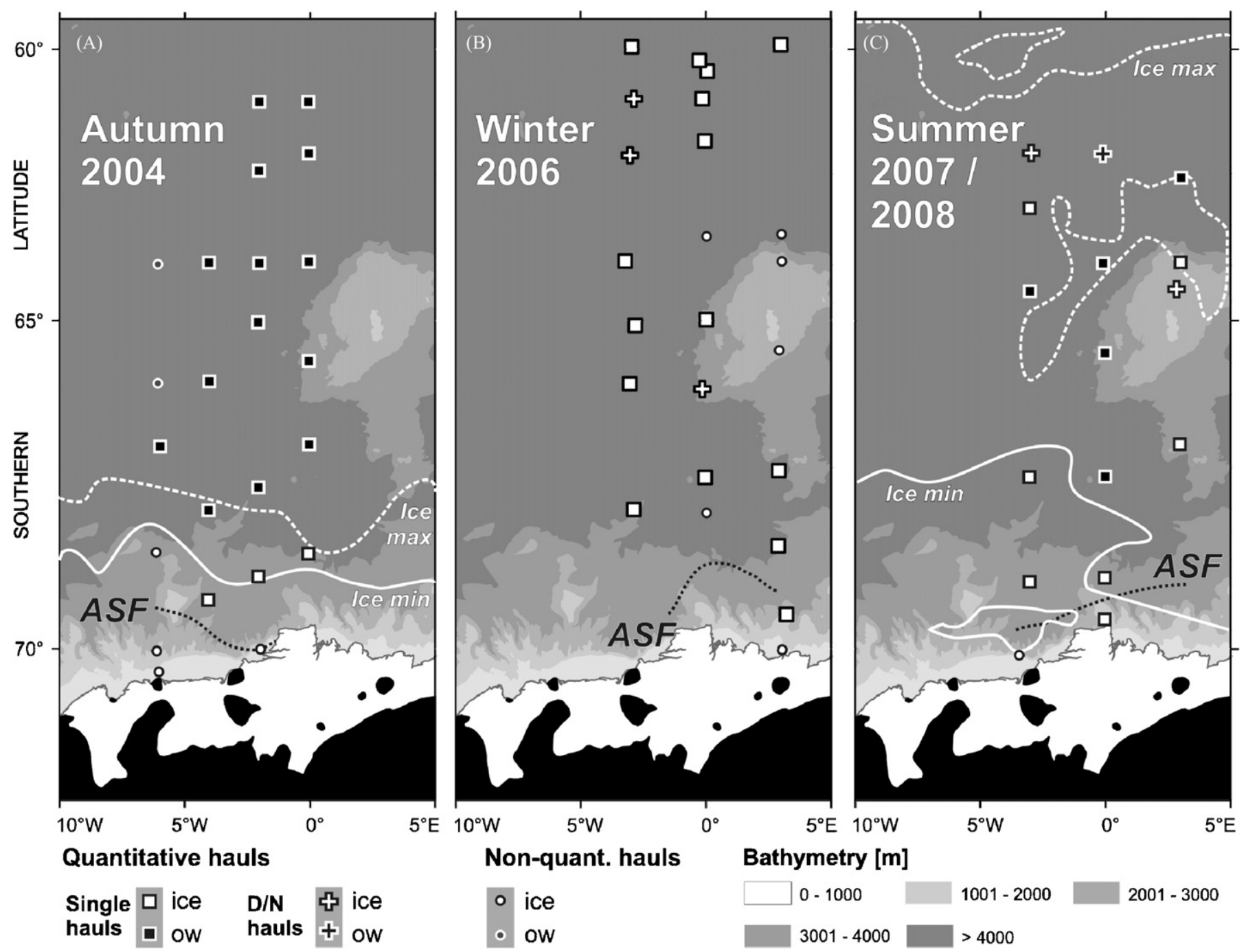

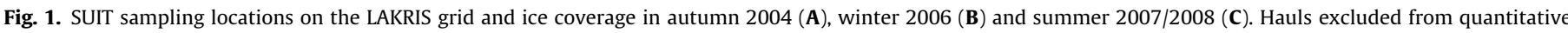

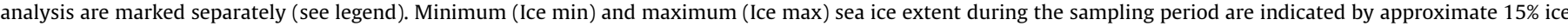

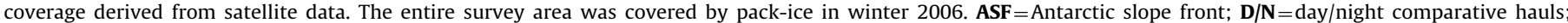
ice $=$ SUIT hauls under ice; $\mathbf{o w}=$ SUIT hauls in open water

Table 1

Technical details of SUIT systems used during LAKRIS expeditions.

\begin{tabular}{|c|c|c|c|c|c|c|}
\hline Expedition & $\begin{array}{l}\text { Mouth } \\
\text { height }\end{array}$ & $\begin{array}{l}\text { Mouth } \\
\text { opening }\end{array}$ & $\begin{array}{l}\text { Main net mesh } \\
\text { size }\end{array}$ & $\begin{array}{l}\text { Plankton net } \\
\text { diameter }\end{array}$ & $\begin{array}{l}\text { Plankton net } \\
\text { mesh size }\end{array}$ & $\begin{array}{l}\text { Additional } \\
\text { equipment }\end{array}$ \\
\hline Autumn 2004 & $225 \mathrm{~cm}$ & $5.06 \mathrm{~m}^{2}$ & $7 \mathrm{~mm}$ & $50 \mathrm{~cm}^{\mathrm{a}}$ & $0.3 \mathrm{~mm}$ & Strobe light, video \\
\hline Winter 2006 & $225 \mathrm{~cm}$ & $5.06 \mathrm{~m}^{2}$ & $7 \mathrm{~mm}$ & ca. $100 \mathrm{~cm}^{\mathrm{b}}$ & $0.3 \mathrm{~mm}$ & Strobe light, $\mathrm{ADCP}$ \\
\hline Summer $2007 / 2008$ & $231 \mathrm{~cm}$ & $4.53 \mathrm{~m}^{2}$ & $7 \mathrm{~mm}$ & ca. $100 \mathrm{~cm}^{\mathrm{b}}$ & $0.3 \mathrm{~mm}$ & $\mathrm{ADCP}$, video \\
\hline
\end{tabular}

a Separate plankton net centered in main net.

${ }^{\mathrm{b}}$ Flexible plankton net sewn into main net.

character of the shrimp net in relation to their slim body shapes. In autumn 2004, ctenophores were not quantified, because they were disrupted by the shrimp net and not caught in sufficient amounts with the small plankton net (Table 1). The large gauzelined codends used in winter 2006 and summer 2007/2008 enabled the collection of intact ctenophores.

\subsection{Data analysis}

Data from the 57 quantitative hauls were used to calculate the arithmetic mean density of individuals of each species
[N $1,000 \mathrm{~m}^{-2}$ ] for different sampling seasons and for hauls conducted under ice and in open water, respectively. Mean wet masses [g $1,000 \mathrm{~m}^{-2}$ ] were computed by multiplication of the abundance of each species with the replacement volume per individual in each season, assuming $1 \mathrm{ml}=1 \mathrm{~g}$. Data were $\log$ $(x+1)$ transformed for comparative ANOVA analysis to obtain similar variances in the residuals.

Patterns in species composition were explored for all samples combined by non-metric multi dimensional scaling (NMDS, (Kruskal, 1964). These analyses were based on a Bray-Curtis dissimilarity matrix produced from the Wisconsin double 
standardized abundance data. In Wisconsin double standardization, species are first standardized by maxima and then sites by site totals (Bray and Curtis, 1957; Oksanen et al., 2008). Ctenophores and taxa not determined to species level, and so possibly representing more than one species, were excluded from community analysis. Statistical analysis was conducted using the $\mathrm{R}$ software package (R 2.7.1, (R-Development-Core-Team, 2008).

\section{Results}

\subsection{Hydrography and ice coverage}

The hydrography of the Lazarev Sea in autumn 2004, winter 2006 and summer 2007/2008 was described in detail by Hunt et al. (in press). The largest part of the LAKRIS survey area was characterized by the inflow of Warm Deep Water (WDW) of circumpolar origin with the southern, westward setting branch of the Weddell Gyre. The Coastal Current south of the Antarctic Slope Front (ASF) was limited to few stations in the very south of the area of investigation in each sampling season (Fig. 1). The mixed layer depth ranged between 12 and $200 \mathrm{~m}$ in autumn 2004, and between 30 and $450 \mathrm{~m}$ in winter 2006, whereas it never exceeded $50 \mathrm{~m}$ in summer $2007 / 2008$.

In autumn 2004, significant amounts of sea ice occurred only south of $68^{\circ} \mathrm{S}$ (Fig. $1 \mathrm{~A}$ ). Most parts of the predominantly young ice cover in that season had formed only days to weeks before the sampling. A substantial pack-ice cover was present throughout the entire area of investigation in winter 2006 (Fig. 1 B). The ice edge during the 2006 sampling period was situated north of the LAKRIS grid at approximately $57^{\circ} \mathrm{S}$. A very dynamic sea ice situation was characteristic of summer 2007/2008. Large parts of the survey area were ice-covered at the beginning of the sampling campaign in early December 2007. A polynia north of Maud Rise expanded during the investigation period, resulting in an intermediate situation with an open area between $63^{\circ} \mathrm{S}$ and $67^{\circ} \mathrm{S}$. When the area was left in late January 2008 , only a residual ice cover persisted south of $67^{\circ} \mathrm{S}$ (Fig. 1C).

\subsection{Species composition, abundance and biomass}

At least 46 macrofauna species were encountered in the upper two metres of the Lazarev Sea, of which 17 were common to all three sampling seasons. The majority of species were crustaceans (17 species) and vertebrates (10 species). Overall species richness was higher in summer 2008 (34 species) compared to autumn 2004 (25 species) and winter 2006 (26 species) (Table 2). With the highest species richness in the sampling season with the lowest number of hauls (summer 2007/2008), an effect of sample size on the differences in species richness between seasons was not evident.

Postlarval Antarctic krill Euphausia superba clearly dominated the species community in terms of both abundance and wet mass in each year (Table 2; Table 3). Next to Antarctic krill, the pteropods Clione antarctica and Clio pyramidata, the amphipods Cyllopus lucasii, Hyperiella dilatata and the arrow worm Sagitta gazellae were abundant in all three seasons. The dominance of postlarval Antarctic krill was less pronounced in summer, when Thysanoessa macrura and S. gazellae corresponded to about $30 \%$ of the mean abundance of Antarctic krill. In open water, T. macrura was the most abundant species in summer (Table 2).

Mean bulk wet mass of macrofauna was highest in winter 2006, followed by autumn 2004 and summer 2007/2008. These differences were largely due to variations in the wet mass density of Antarctic krill in each sampling season (Fig. 5). When data were split by ice-covered and open water stations, a seasonally divergent pattern was apparent between autumn and summer. In autumn, mean wet mass of all species including postlarval Antarctic krill was very low under ice, whereas it was dominated by about two orders of magnitude higher values in Antarctic krill in open waters. In summer, Antarctic krill clearly dominated in both abundance and wet mass under ice. In open waters, ctenophores accounted for more than $60 \%$ of the average wet mass (Table 3).

\subsection{Seasonality, effect of sea ice and diel patterns}

NDMS plots were used to visualize the community structure. Fig. 3 shows an NMDS plot based on the species abundance data from all 3 sampling seasons. The relatively homogeneous distribution of sampling stations in the NMDS plot yielded no evidence for the presence of distinct communities. A pronounced seasonal pattern in the community structure, however, was indicated by a gradual ordination of samples according to sampling season (Fig. 3). Accordingly, the horizontal ordination axis 1 was highly correlated with the sampling season (Pearson correlation coefficient: 0.97 ; $<<<0.001$ ). In addition, a relationship of the presence of sea ice during SUIT hauls with the sample ordination was apparent from a correlation of NMDS ordination axis 2 with the presence of sea ice (Pearson correlation coefficient: $0.91 ; \mathrm{p}<<0.001$; Fig. 3 ).

The impact of sampling season and sea ice on species composition was investigated more closely at the level of diversity indices and a selection of dominant species. Average species richness significantly responded to both factors and was highest in summer 2007/2008, lowest in autumn 2004, and was generally higher in open water than at sea ice stations. This pattern of elevated diversity in summer and in open water, however, was not significantly reflected in the Shannon index (Fig. 4; Table 4). A detailed analysis of the abundance, distribution and population structure of surface layer euphausiids from the present data collection, including a description of their relationship with the presence of sea ice at different seasons, was provided by Flores et al. (2009). Among non-euphausiid species, Clio pyramidata, Eusirus microps and Sagitta gazellae were significantly more abundant in summer than in autumn and winter (Fig. 5A, D, H; Table 4). The opposite trend was apparent in Eukrohnia hamata (Fig. 5G; Table 4). In addition to season, the presence of sea ice had a significant negative effect on the abundance of Cyllopus lucasii (Fig. 5E; Table 4). A preference for open water was also significant in Hyperiella dilatata, independent of the sampling season (Fig. 5F; Table 4). It was not possible to separate the effects of season and the presence of sea ice from each other in Clione antarctica and Eusirus laticarpus due to a significant interaction of the two factors (Table 4). While the highest average abundance of C. antarctica was under the winter pack-ice, the pteropod was more abundant in open water than under ice in autumn and summer (Fig. 5B). E. laticarpus was not encountered in autumn. It was significantly more abundant under ice than in open waters in summer. The mean open water abundance in summer, however, was still above under-ice densities in winter 2006, when no open water stations were sampled for comparison.

A significant diel influence on the species composition in winter 2006 and summer 2007/2008 was apparent from a number of abundant species at the five stations repeatedly sampled at day and night. In winter 2006, Clione antarctica was clearly abundant in the ice-water interface layer at night and largely absent from this environment at day (Fig. 6A). A similar, but less pronounced pattern was apparent in Eukrohnia hamata at the only station where enough animals were caught (Fig. 6B). At the 3 stations sampled in summer 2007/2008, these two species 
Table 2

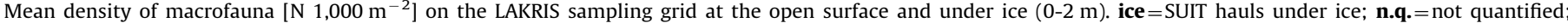
$\mathbf{o w}=$ SUIT hauls in open water

\begin{tabular}{|c|c|c|c|c|c|c|c|}
\hline & \multicolumn{3}{|l|}{$\begin{array}{l}\text { Autumn } \\
2004\end{array}$} & \multirow{2}{*}{$\begin{array}{l}\text { Winter } 2006 \\
\text { ice (total) }\end{array}$} & \multicolumn{3}{|c|}{ Summer $2007 / 2008$} \\
\hline & ow & ice & total & & ow & ice & total \\
\hline \multicolumn{8}{|l|}{ CNIDARIA } \\
\hline Calycopsis borchgrevinki & 0.033 & 0.000 & 0.027 & 0.097 & 0.000 & 0.000 & 0.000 \\
\hline Diphyes antarctica & 0.954 & 0.088 & 0.802 & 21.918 & 0.000 & 0.018 & 0.011 \\
\hline \multicolumn{8}{|l|}{ CTENOPHORA } \\
\hline Beroida & n.q. & n.q. & n.q. & 0.194 & 0.261 & 0.000 & 0.101 \\
\hline Beroe cucumis & n.q. & n.q. & n.q. & n.q. & 0.692 & 0.174 & 0.375 \\
\hline Beroe forskalii & n.q. & n.q. & n.q. & n.q. & 2.327 & 0.131 & 0.985 \\
\hline \multicolumn{8}{|l|}{ Cydipidda } \\
\hline Callianira antarctica & n.q. & n.q. & n.q. & n.q. & 1.130 & 0.344 & 0.650 \\
\hline \multicolumn{8}{|l|}{$\begin{array}{l}\text { MOLLUSCA } \\
\text { Pteropoda }\end{array}$} \\
\hline Clione antarctica & 0.094 & 0.000 & 0.077 & 4.199 & 2.636 & 0.604 & 1.394 \\
\hline Clio piatkowskii & 0.000 & 0.000 & 0.000 & 0.000 & 0.104 & 0.000 & 0.041 \\
\hline C. pyramidata & 2.084 & 0.464 & 1.798 & 0.702 & 47.411 & 9.086 & 23.990 \\
\hline Spongiobranchaea australis & 0.008 & 0.182 & 0.039 & 0.072 & 0.035 & 0.000 & 0.014 \\
\hline \multicolumn{8}{|l|}{ Cephalopoda } \\
\hline $\begin{array}{l}\text { Paralarvae } \\
\text { Psychroteuthis glacialis } \\
\text { Adults }\end{array}$ & 0.000 & 0.000 & 0.000 & 0.000 & 0.140 & 0.044 & 0.082 \\
\hline Kondakovia longimana & 0.000 & 0.000 & 0.000 & 0.012 & 0.000 & 0.000 & 0.000 \\
\hline \multicolumn{8}{|l|}{$\begin{array}{l}\text { ANNELIDA } \\
\text { Polychaeta }\end{array}$} \\
\hline Tomopteris carpenteri & 0.083 & 0.000 & 0.068 & 0.065 & 1.427 & 0.635 & 0.943 \\
\hline T. planctonis & 0.000 & 0.000 & 0.000 & 0.000 & 0.209 & 0.593 & 0.443 \\
\hline T. septentrionalis & 0.162 & 0.000 & 0.134 & 0.025 & 0.363 & 0.019 & 0.153 \\
\hline Vanadis antarctica & 0.161 & 0.000 & 0.133 & 0.042 & 0.000 & 0.018 & 0.011 \\
\hline \multicolumn{8}{|l|}{$\begin{array}{l}\text { ARTHROPODA } \\
\text { Crustacea } \\
\text { Amphipoda }\end{array}$} \\
\hline Eusirus laticarpus & 0.000 & 0.000 & 0.000 & 0.294 & 0.732 & 9.926 & 6.351 \\
\hline E. microps & 0.000 & 0.000 & 0.000 & 0.118 & 0.280 & 0.487 & 0.406 \\
\hline Ischyrocerus sp. & 0.000 & 0.000 & 0.000 & 0.050 & 0.000 & 0.000 & 0.000 \\
\hline Scina sp. & 0.000 & 0.000 & 0.000 & 0.015 & 0.000 & 0.000 & 0.000 \\
\hline Cyllopus lucasii & 6.423 & 0.000 & 5.290 & 0.119 & 2.070 & 0.000 & 0.805 \\
\hline Hyperia macrocephala & 0.054 & 0.000 & 0.044 & 0.025 & 0.035 & 0.133 & 0.095 \\
\hline Hyperiella antarctica & 0.290 & 0.000 & 0.239 & 0.000 & 0.283 & 0.000 & 0.110 \\
\hline Hyperiella dilatata & 2.810 & 0.000 & 2.314 & 0.088 & 3.781 & 0.039 & 1.494 \\
\hline Hyperiella macronyx & 0.035 & 0.000 & 0.029 & 0.000 & 0.283 & 0.000 & 0.110 \\
\hline Hyperoche capucinus & 1.015 & 0.265 & 0.883 & 0.202 & 0.000 & 0.000 & 0.000 \\
\hline Hyperoche medusarum & 0.000 & 0.000 & 0.000 & 0.000 & 0.246 & 0.083 & 0.147 \\
\hline Primno macropa & 0.000 & 0.000 & 0.000 & 0.172 & 0.876 & 0.883 & 0.880 \\
\hline \multicolumn{8}{|l|}{ Euphausiacea } \\
\hline Euphausia crystallorophias & 0.000 & 0.000 & 0.000 & 0.000 & 0.000 & 0.125 & 0.076 \\
\hline E frigida & 0.926 & 0.000 & 0.763 & 0.000 & 0.000 & 0.000 & 0.000 \\
\hline E. superba & 932.734 & 6.025 & 769.197 & 2392.755 & 94.623 & 1100.468 & 709.306 \\
\hline Thysanoessa macrura & 0.000 & 0.000 & 0.000 & 0.000 & 164.985 & 240.651 & 211.225 \\
\hline \multicolumn{8}{|l|}{ Decapoda (larvae) } \\
\hline Unidentified decapod & 0.000 & 0.000 & 0.000 & 0.000 & 0.138 & 0.134 & 0.135 \\
\hline CHAETOGNATHA & & & & & & & \\
\hline Eukrohnia hamata & 5.383 & 1.402 & 4.680 & 3.282 & 0.133 & 0.000 & 0.052 \\
\hline Sagitta gazellae & 1.499 & 1.293 & 1.462 & 2.745 & 42.126 & 23.236 & 30.582 \\
\hline $\begin{array}{l}\text { CHORDATA } \\
\text { Salpida }\end{array}$ & & & & & & & \\
\hline Ihea racovitzai & 0.072 & 0.182 & 0.091 & 0.000 & 0.000 & 0.049 & 0.030 \\
\hline Salpa thompsoni & 0.063 & 0.000 & 0.052 & 0.819 & 0.600 & 0.319 & 0.428 \\
\hline Vertebrata & & & & & & & \\
\hline Fish larvae & & & & & & & \\
\hline Dissostichus sp. & 0.000 & 0.000 & 0.000 & 0.000 & 0.035 & 0.000 & 0.014 \\
\hline Lepidonotothen squamifrons & 0.026 & 0.000 & 0.022 & 0.000 & 0.000 & 0.000 & 0.000 \\
\hline Pagothenia borchgrevinki & 0.000 & 0.000 & 0.000 & 0.025 & 0.000 & 0.000 & 0.000 \\
\hline Trematomus loennbergii & 0.021 & 0.000 & 0.018 & 0.234 & 0.000 & 0.043 & 0.026 \\
\hline T. scotti & 0.010 & 0.000 & 0.008 & 0.000 & 0.000 & 0.000 & 0.000 \\
\hline Gymnodraco acuticeps & 0.000 & 0.077 & 0.014 & 0.000 & 0.000 & 0.000 & 0.000 \\
\hline Unidentified channichthyid & 0.000 & 0.000 & 0.000 & 0.000 & 0.000 & 0.025 & 0.015 \\
\hline
\end{tabular}




\begin{tabular}{|c|c|c|c|c|c|c|c|}
\hline & \multicolumn{3}{|l|}{$\begin{array}{l}\text { Autumn } \\
2004\end{array}$} & \multirow{2}{*}{$\begin{array}{l}\text { Winter } 2006 \\
\text { ice (total) }\end{array}$} & \multicolumn{3}{|c|}{ Summer $2007 / 2008$} \\
\hline & ow & ice & total & & ow & ice & total \\
\hline \multicolumn{8}{|l|}{ Postlarval fish } \\
\hline Notolepis sp. (juveniles) & 0.000 & 0.000 & 0.000 & 0.000 & 0.136 & 0.019 & 0.064 \\
\hline Electrona antarctica & 0.064 & 0.000 & 0.053 & 0.013 & 0.000 & 0.000 & 0.000 \\
\hline Aethotaxis mitopteryx & 0.000 & 0.000 & 0.000 & 0.012 & 0.000 & 0.000 & 0.000 \\
\hline Total & 955.004 & 9.980 & 788.235 & 2428.293 & 368.098 & 1388.286 & 991.546 \\
\hline Species Richness & 24 & 9 & 25 & 27 & 29 & 27 & 35 \\
\hline
\end{tabular}

Table 3

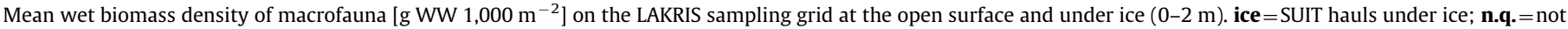
quantified; ow $=$ SUIT hauls in open water

\begin{tabular}{|c|c|c|c|c|c|c|c|}
\hline & \multicolumn{3}{|c|}{ Autumn 2004} & \multirow{2}{*}{$\begin{array}{l}\text { Winter } 2006 \\
\text { ice (total) }\end{array}$} & \multicolumn{3}{|c|}{ Summer 2007/2008 } \\
\hline & ow & ice & total & & ow & ice & total \\
\hline \multicolumn{8}{|l|}{ CNIDARIA } \\
\hline Calycopsis borchgrevinki & 0.033 & 0.000 & 0.027 & 0.342 & 0.000 & 0.000 & 0.000 \\
\hline Diphyes antarctica & 0.280 & 0.026 & 0.235 & 6.426 & 0.000 & 0.005 & 0.003 \\
\hline \multicolumn{8}{|l|}{ CTENOPHORA } \\
\hline \multicolumn{8}{|l|}{ Beroida } \\
\hline Beroe cucumis & n.q. & n.q. & n.q. & n.q. & 11.556 & 2.907 & 6.271 \\
\hline \multicolumn{8}{|l|}{ Cydipidda } \\
\hline Callianira antarctica & n.q. & n.q. & n.q. & n.q. & 8.217 & 2.503 & 4.725 \\
\hline \multicolumn{8}{|l|}{ MOLLUSCA } \\
\hline Clione antarctica & 0.038 & 0.000 & 0.031 & 0.897 & 0.573 & 0.132 & 0.303 \\
\hline Clio piatkowskii & 0.000 & 0.000 & 0.000 & 0.000 & 0.174 & 0.000 & 0.068 \\
\hline C. pyramidata & 0.761 & 0.170 & 0.657 & 0.035 & 12.758 & 2.445 & 6.456 \\
\hline Spongiobranchaea australis & 0.001 & 0.023 & 0.005 & 0.009 & 0.004 & 0.000 & 0.002 \\
\hline \multicolumn{8}{|l|}{ Cephalopoda } \\
\hline $\begin{array}{l}\text { Paralarvae } \\
\text { Psychroteuthis glacialis } \\
\text { Adults }\end{array}$ & 0.000 & 0.000 & 0.000 & 0.000 & 0.094 & 0.029 & 0.054 \\
\hline Kondakovia longimana & 0.000 & 0.000 & 0.000 & 2.877 & 0.000 & 0.000 & 0.000 \\
\hline \multicolumn{8}{|l|}{$\begin{array}{l}\text { ANNELIDA } \\
\text { Polychaeta }\end{array}$} \\
\hline Tomopteris carpenteri & 0.086 & 0.000 & 0.071 & 0.067 & 1.484 & 0.660 & 0.980 \\
\hline T. planctonis & 0.000 & 0.000 & 0.000 & 0.000 & 0.278 & 0.251 & 0.188 \\
\hline T. septentrionalis & 0.069 & 0.000 & 0.057 & 0.033 & 0.484 & 0.026 & 0.204 \\
\hline Vanadis antarctica & 0.161 & 0.000 & 0.133 & 0.042 & 0.000 & 0.018 & 0.011 \\
\hline \multicolumn{8}{|l|}{$\begin{array}{l}\text { ARTHROPODA } \\
\text { Crustacea } \\
\text { Amphipoda }\end{array}$} \\
\hline Eusirus laticarpus & 0.000 & 0.000 & 0.000 & 0.029 & 0.072 & 0.980 & 0.627 \\
\hline E. microps & 0.000 & 0.000 & 0.000 & 0.098 & 0.119 & 0.206 & 0.172 \\
\hline Ischyrocerus sp. & 0.000 & 0.000 & 0.000 & 0.011 & 0.000 & 0.000 & 0.000 \\
\hline Scina sp. & 0.000 & 0.000 & 0.000 & 0.000 & 0.000 & 0.000 & 0.000 \\
\hline Cyllopus lucasii & 1.686 & 0.000 & 1.389 & 0.030 & 0.517 & 0.000 & 0.201 \\
\hline Hyperia macrocephala & 0.051 & 0.000 & 0.042 & 0.024 & 0.033 & 0.126 & 0.089 \\
\hline Hyperiella antarctica & 0.018 & 0.000 & 0.015 & 0.000 & 0.018 & 0.000 & 0.007 \\
\hline Hyperiella dilatata & 0.176 & 0.000 & 0.145 & 0.000 & 0.236 & 0.002 & 0.093 \\
\hline Hyperiella macronyx & 0.002 & 0.000 & 0.002 & 0.000 & 0.018 & 0.000 & 0.007 \\
\hline Hyperoche capucinus & 0.184 & 0.048 & 0.160 & 0.067 & 0.000 & 0.000 & 0.000 \\
\hline Hyperoche medusarum & 0.000 & 0.000 & 0.000 & 0.000 & 0.062 & 0.021 & 0.037 \\
\hline Primno macropa & 0.000 & 0.000 & 0.000 & 0.230 & 0.088 & 0.088 & 0.088 \\
\hline \multicolumn{8}{|l|}{ Euphausiacea } \\
\hline Euphausia crystallorophias & 0.000 & 0.000 & 0.000 & 0.000 & 0.000 & 0.007 & 0.004 \\
\hline E frigida & 0.041 & 0.000 & 0.034 & 0.000 & 0.000 & 0.000 & 0.000 \\
\hline E. superba & 335.295 & 2.166 & 276.507 & 545.917 & 14.804 & 172.175 & 110.975 \\
\hline Thysanoessa macrura & 0.000 & 0.000 & 0.000 & 0.000 & 7.937 & 11.577 & 10.162 \\
\hline \multicolumn{8}{|l|}{ Decapoda (larvae) } \\
\hline Unidentified decapod & 0.000 & 0.000 & 0.000 & 0.000 & 0.008 & 0.008 & 0.008 \\
\hline \multicolumn{8}{|l|}{ CHAETOGNATHA } \\
\hline Eukrohnia hamata & 0.094 & 0.025 & 0.082 & 0.057 & 0.002 & 0.000 & 0.001 \\
\hline Sagitta gazellae & 0.244 & 0.210 & 0.238 & 0.634 & 22.428 & 12.371 & 16.282 \\
\hline
\end{tabular}




\begin{tabular}{|c|c|c|c|c|c|c|c|}
\hline & \multicolumn{3}{|c|}{ Autumn 2004} & \multirow{2}{*}{$\begin{array}{l}\text { Winter } 2006 \\
\text { ice (total) }\end{array}$} & \multicolumn{3}{|c|}{ Summer 2007/2008 } \\
\hline & ow & ice & total & & ow & ice & total \\
\hline \multicolumn{8}{|l|}{$\begin{array}{l}\text { CHORDATA } \\
\text { Salpida }\end{array}$} \\
\hline Ihea racovitzai & 0.108 & 0.273 & 0.137 & 0.000 & 0.000 & 0.074 & 0.045 \\
\hline Salpa thompsoni & 0.058 & 0.000 & 0.048 & 1.281 & 0.948 & 0.504 & 0.676 \\
\hline \multicolumn{8}{|l|}{$\begin{array}{l}\text { Vertebrata } \\
\text { Fish larvae }\end{array}$} \\
\hline Dissostichus sp. & 0.000 & 0.000 & 0.000 & 0.000 & 0.035 & 0.000 & 0.014 \\
\hline Lepidonotothen squamifrons & 0.026 & 0.000 & 0.022 & 0.000 & 0.000 & 0.000 & 0.000 \\
\hline Pagothenia borchgrevinki & 0.000 & 0.000 & 0.000 & 0.025 & 0.000 & 0.000 & 0.000 \\
\hline Trematomus loennbergii & 0.008 & 0.000 & 0.007 & 0.090 & 0.000 & 0.017 & 0.010 \\
\hline T. scotti & 0.010 & 0.000 & 0.008 & 0.000 & 0.000 & 0.000 & 0.000 \\
\hline Gymnodraco acuticeps & 0.000 & 0.077 & 0.014 & 0.000 & 0.000 & 0.000 & 0.000 \\
\hline Unidentified channichthyid & 0.000 & 0.000 & 0.000 & 0.000 & 0.000 & 0.025 & 0.015 \\
\hline \multicolumn{8}{|l|}{ Postlarval fish } \\
\hline Notolepis sp. (juveniles) & 0.000 & 0.000 & 0.000 & 0.000 & 0.122 & 0.017 & 0.058 \\
\hline Electrona antarctica & 0.135 & 0.000 & 0.111 & 0.013 & 0.000 & 0.000 & 0.000 \\
\hline Aethotaxis mitopteryx & 0.000 & 0.000 & 0.000 & 0.312 & 0.000 & 0.000 & 0.000 \\
\hline Total & 339.564 & 3.018 & 280.174 & 560.182 & 233.742 & 215.493 & 222.511 \\
\hline
\end{tabular}

Table 4

Relationship of a selection of non-euphausiid zooplankton species, species richness and Shannon diversity index from the surface layer $(0-2 \mathrm{~m})$ with the presence of sea ice, sampling season and the interaction of the 2 factors. Significance (ANOVA): ${ }^{*} \mathrm{p}<0.05 ;{ }^{* *} \mathrm{p}<0.01 ;{ }^{* * *} P<0.001 ;$ ns $=$ not significant. Corresponding euphausiid data were presented in Flores et al. (2009).

\begin{tabular}{llll}
\hline & $\begin{array}{l}\text { Presence } \\
\text { of sea } \text { ice }\end{array}$ & Season & $\begin{array}{c}\text { Interaction } \\
\text { (ice : season) }\end{array}$ \\
\hline Clio pyramidata & $\mathrm{ns}$ & $* * *$ & $\mathrm{~ns}$ \\
Clione antarctica & $*$ & $\mathrm{~ns}$ & $* * *$ \\
Eusirus laticarpus & $* * *$ & $* * *$ & $* * *$ \\
E. microps & $\mathrm{ns}$ & $* *$ & $\mathrm{~ns}$ \\
Cyllopus lucasii & $* * *$ & $*$ & $\mathrm{~ns}$ \\
Hyperiella dilatata & $* * *$ & $\mathrm{~ns}$ & $\mathrm{~ns}$ \\
Eukrohnia hamata & $\mathrm{ns}$ & $* * *$ & $\mathrm{~ns}$ \\
Sagitta gazellae & $\mathrm{ns}$ & $* * *$ & $\mathrm{~ns}$ \\
Species richness & $* *$ & & $\mathrm{~ns}$ \\
Shannon index & $\mathrm{ns}$ & $\mathrm{ns}$ & $\mathrm{ns}$ \\
\hline
\end{tabular}

were not sufficiently abundant to allow meaningful diel comparisons. In this season, Clio pyramidata (Fig. 7A), Cyllopus lucasii (Fig. 7C), Hyperiella dilatata (Fig. 7D) and Sagitta gazellae (Fig. 7E) were abundant at night, but largely absent from the surface layer during day. This pattern was independent of the presence of sea ice in $C$. pyramidata and $S$. gazellae. The other two species were not $(C$. lucasii) or barely ( $H$. dilatata) encountered under ice. A pronounced diel pattern was not apparent in Eusirus laticarpus, which was twice as abundant at day as at night at one of the diel comparative stations sampled under ice, and almost equally abundant at the other. Only a few specimens were caught at night at the open water station (Fig. 7B).

\section{Discussion}

\subsection{Taxonomic composition and diversity}

The taxonomic range of macrozooplankton and micronekton found in the 0-2 $\mathrm{m}$ surface layer covered 7 phyla ranging from hydrozoans to vertebrates, included free drifting, actively swimming and potentially sympagic species ranging in size order from millimeters (Hyperiella spp.) to decimeters (squid, fish) (Table 2).
Variability in community composition was related to sampling season and the presence of sea ice (Fig. 2). This variability was reflected in a higher average species richness in summer 2007/ 2008 compared to autumn 2004 and winter 2006, and a generally lower average species richness under sea ice than in the open surface layer (Fig. 4).

Zooplankton community composition can vary considerably depending on sampling methodology and effort, sampling depth, seasonal and geographical coverage. Like in all fishing nets, the selectivity of the SUIT depends on the towing speed, the size of the net opening, the mesh size, the size and the body shape of the targeted species and their ability to detect and actively avoid the net. Our range of towing speed of 1.5-2.5 knots was in the range commonly used in macrozooplankton studies (Donnelly et al., 2006; Fisher et al., 2004). ADCP flow measurements indicated that factors potentially causing differences in catch efficiency between under-ice and open water sampling, such as turbulences caused by waves or troughs and ridges under the ice, were not sufficient to account for the almost 3-fold difference in abundance between under-ice and open water samples in summer 2007/2008 (Table 2; van Franeker et al., 2009).

Based on the mesh size of the nets used $(7 \mathrm{~mm})$, it can be assumed that hard-bodied zooplankton was captured quantitatively down to a minimum size of $10 \mathrm{~mm}$, and only minor losses can be expected in 5 to $10 \mathrm{~mm}$ sized species. Slim-bodied species, such as copepods and euphausiid furcilia larvae, may have slipped through the meshes of the shrimp net to a larger extent and were therefore excluded from the analysis. A comparison of the size composition of Antarctic krill Euphausia superba between catches of SUIT and the well-established rectangular midwater trawl (RMT) at identical sampling stations indicated that the size selectivity of the SUIT did not differ significantly from the RMT at least up to the size of large euphausiids (Flores et al., 2009). Because swimming speed and thus escape capability in postlarval Antarctic krill is related to size (Kils, 1979), this similarity in size distribution indicates that SUIT catches were not more affected by size-dependent net avoidance than conventional micronekton trawls. The true extent of net avoidance in different species and size classes, however, can only be quantified by a dedicated methodological study. To date, SUIT provides the most quantitative way to directly sample macrozooplankton and micronekton in the ice-water interface layer and in the open surface layer with the same gear. This approach overcomes the spatial limitation of 


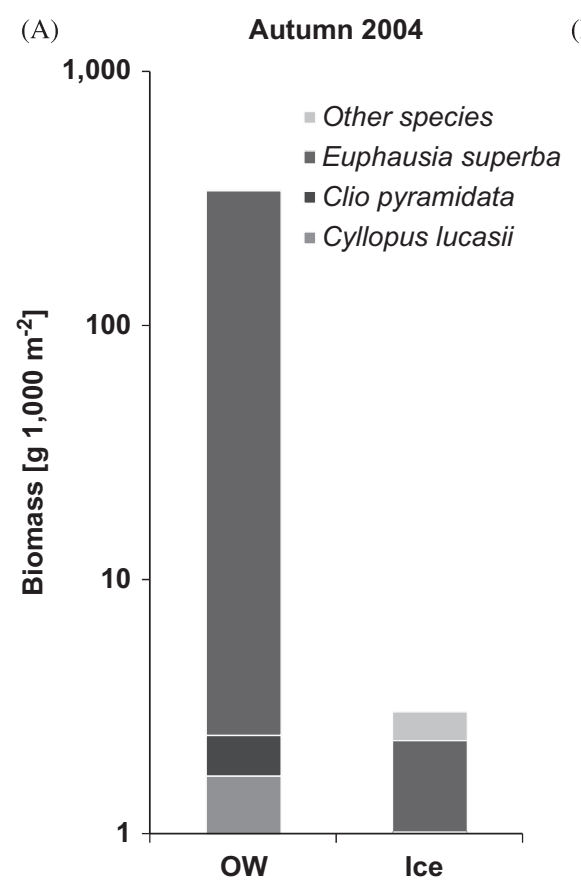

(B)

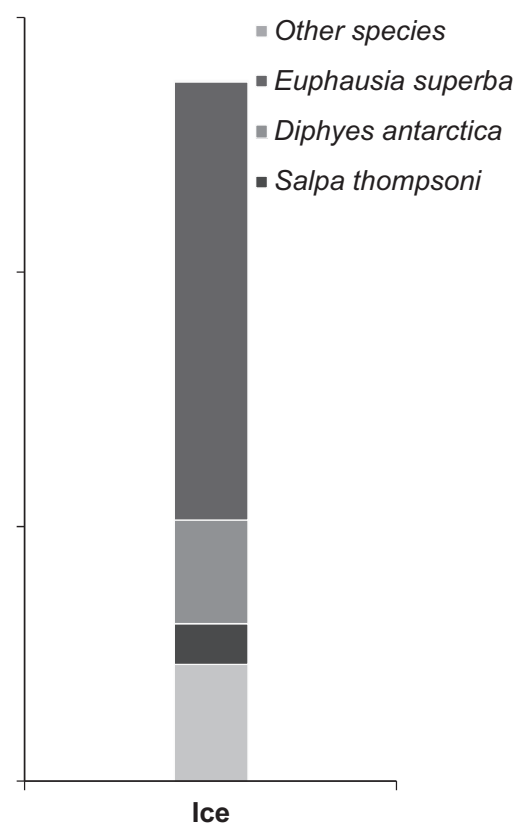

(C)

Summer 2007 / 2008

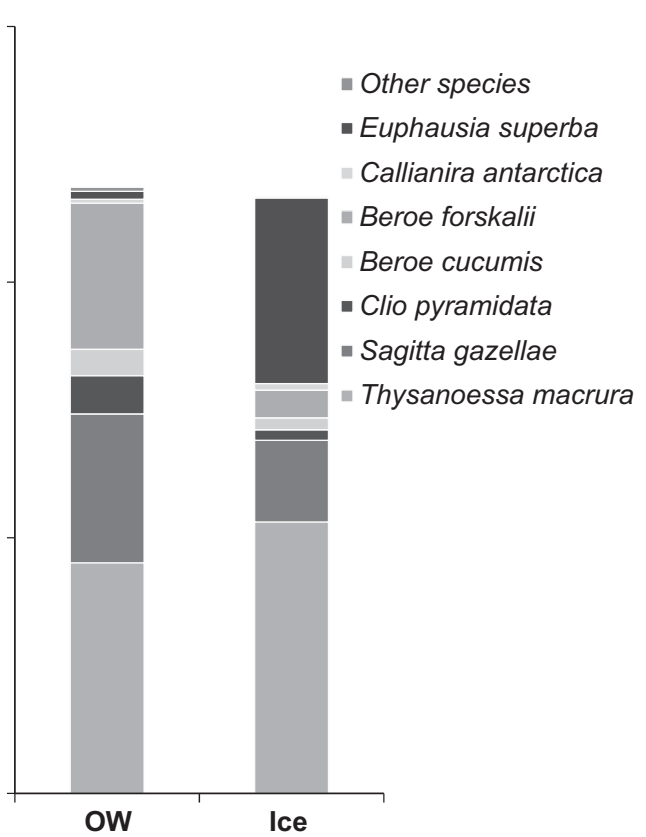

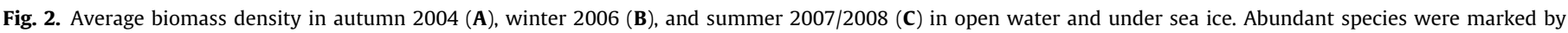
different shades of gray in each sampling season. ice =SUIT hauls under ice; ow=SUIT hauls in open water.

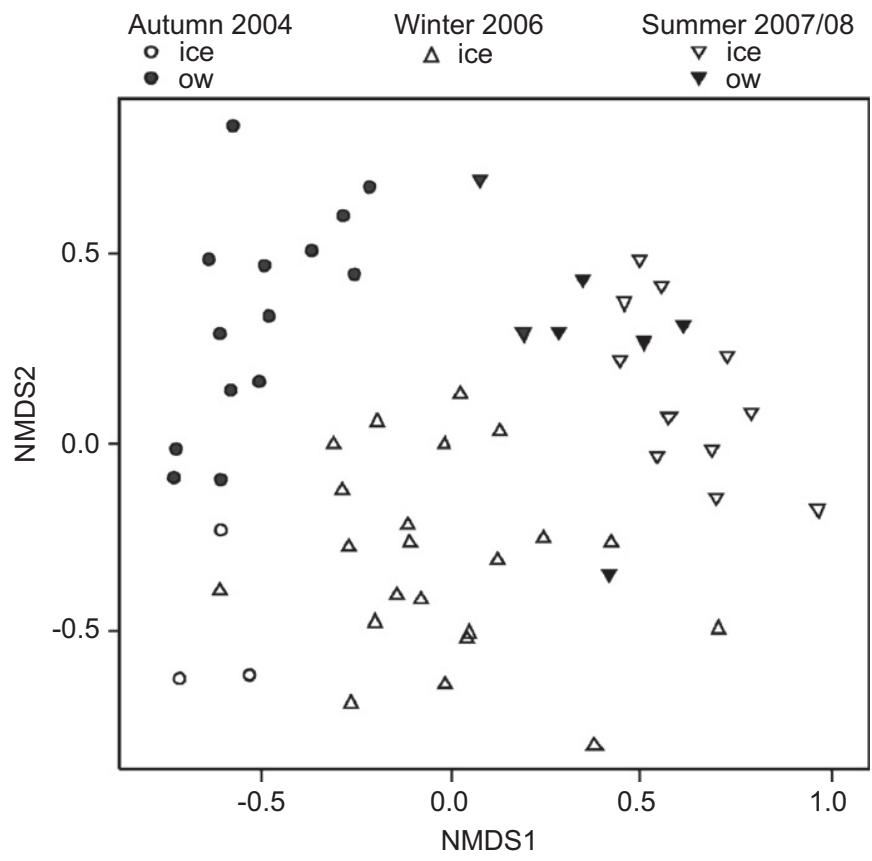

Fig. 3. NMDS plot of SUIT samples. Samples were marked according to sampling season and under-ice/open water hauls. ice =SUIT hauls under ice; ow $=$ SUIT hauls in open water; NMDS 1, 2 =ordination axes.

observations by divers and autonomous camera systems and the problems with surface reflections and accurate determination of species and their size compositions associated with hydroacoustic methods (Brierley et al., 2002; Cisewski et al., 2010; Kaufmann et al., 1995; Marschall, 1988; Zhou et al., 1994). Absolute certainty about the comparability of this new field sampling technique, however, can only slowly be obtained when more comparative datasets become available.
We found 46 macrofauna species in the $0-2 \mathrm{~m}$ surface layer of the Lazarev Sea. Although equal or higher in sample size than the present investigation, many offshore studies in the Southern Ocean with a sampling depth of 50 to $300 \mathrm{~m}$ did not find more than the approximately 30 macrozooplankton/micronekton species per season collected during our study (Donnelly et al., 2006; Fisher et al., 2004; Hunt et al., 2007; Lancraft et al., 1989). Higher species richness was reported from typically more diverse areas, such as the Weddell-Scotia Confluence (Lancraft et al., 1991; Siegel et al., 1992) and the Polar Frontal Zone (Pakhomov and Froneman, 2000), or where substantial parts of the sampling area were on the shelf (Boysen-Ennen and Piatkowski, 1988; Piatkowski, 1989). In spite of its position at the outermost margin of the pelagic realm, the surface layer seems to be barely less diverse than orders of magnitudes greater depth layers in the Antarctic sea ice zone.

The community composition of the surface layer exhibited considerable seasonal variability (Fig. 3). Some seasonal variation is typical for the zooplankton composition of Southern Ocean zooplankton (Fisher et al., 2004; Hunt et al., 2007). Based on data collected largely during the identical surveys, however, Hunt et al. (in press) found only moderate seasonal differences in the community composition of the $0-200 \mathrm{~m}$ depth layer in the Lazarev Sea. The pronounced seasonal signal in community composition seems to be limited to the surface layer. This seasonal variability can partly be attributed to seasonal shifts in the depth distribution towards the surface in summer and to greater depth in winter (Cisewski et al., 2010). A shift of species into the surface layer in summer is also reflected in the higher species richness in summer compared to autumn and winter (Fig. 4).

Average species richness was significantly higher in the open surface layer than under ice (Fig. 5, Table 4). A general trend of decreasing species richness from open water into the closed packice was also observed by Siegel et al. (1992) in the upper $60 \mathrm{~m}$ and, to a lesser extent, by Donnelly et al. (2006) in the 0-200 m stratum. In contrast to the pattern observed in species richness, Shannon diversity under ice was not significantly lower than at 
(A)

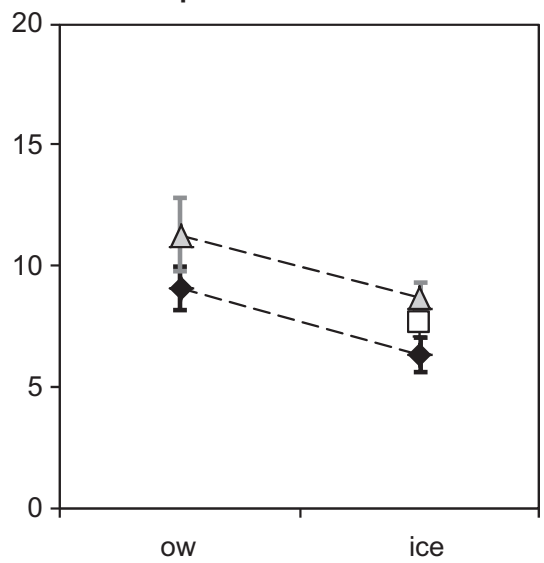

(B)

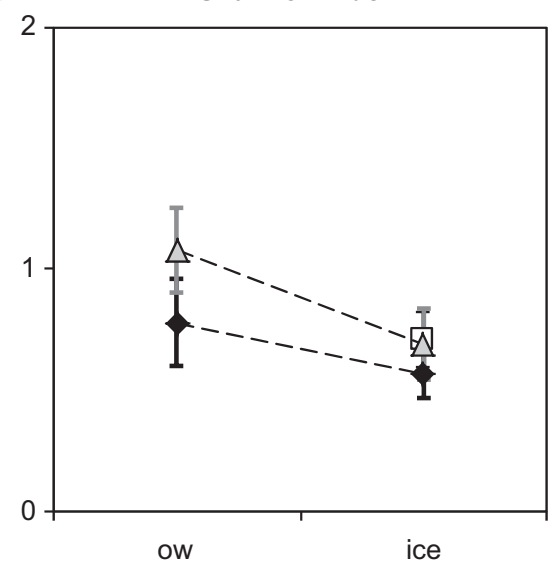

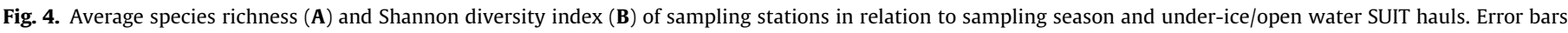
denote standard errors. Dashed line indicates relationship between open water and under-ice samples. Symbols as indicated in Fig. 3.

the open surface in our data from the Lazarev Sea (Fig. 5, Table 4). This index increases with greater evenness and increasing number of rare species, emphasizing the importance of the ice-water interface layer for less abundant species, of which some may be closely associated with the sea ice habitat. This implies that differences in the species richness between the open and icecovered surface layer possibly level off when sampling effort is increased.

\subsection{Ecological significance of the under-ice and open surface layer}

The higher trophic levels of the Antarctic seasonal sea ice zone may depend considerably on energy ultimately derived from the primary production of algae growing in sea ice (Ainley et al., 1991; Ainley et al., 2003; van Franeker et al., 1997). Energy can be transferred from the sea ice community to the pelagic food web through vertical migration, food chains and sinking detritus (reviewed by Brierley and Thomas, 2002). Thus, potentially all biota directly or indirectly depending on sea ice production may act as energy vectors between the ice and the water column.

Among the three seasons sampled during the present study, the overall average wet mass density of non-gelatinous macrozooplankton was highest under the winter sea ice. In summer, it was significantly higher under ice than in the open surface layer. Only in autumn, the average wet mass at the 3 stations sampled under sea ice was negligible compared to open water values (Fig. 2). The young autumn ice had probably not yet developed a rich enough biological community to be attractive for sea ice foragers (Eicken, 1992). It can be concluded that non-gelatinous macrozooplankton biomass in the surface layer is significantly elevated under sea ice in the Lazarev Sea throughout most of the year. High concentrations of biomass under sea ice emphasize the role of the ice-water interface layer as an important site of energy transfer from the sea ice into the pelagic food web (Brierley and Thomas, 2002).

Antarctic krill dominated the wet mass composition in each sampling season, and especially under sea ice, in winter 2006 and summer 2007/2008 (Table 3). Both in terms of wet mass and abundance, Antarctic krill can be considered a key energy vector of the pelagic food web. A separate study therefore focuses on the euphausiids from the Lazarev Sea, allowing a closer look on their horizontal and vertical distribution, population structure and ecological significance. Both in summer and winter, Antarctic krill was often considerably more abundant in the ice-water interface layer than in the underlying water column, indicating that sea ice plays a pivotal role in the functional ecology of this species (Flores et al., 2009).

Besides the often dominant Antarctic krill, other species may directly, indirectly or temporarily be important for the energy transfer between sea ice biota and the pelagic food web. Our results implied a marked response of the species community to the presence of sea ice (Fig. 3; Fig. 5). There was not sufficient evidence, however, to identify distinct communities. Indications for an association with the ice-water interface layer were present in three species: Eusirus laticarpus, E. microps and Clione antarctica. In the latter species, the highest abundances in all three seasons were encountered under the sea ice in winter 2006. In autumn and summer, however, abundances were generally higher in water than under ice, causing a significant interaction of the factors season and ice-presence (Fig. 5; Table 4). Because no stations were sampled in open water in 2006, we cannot exclude that the high densities under winter ice reflected inter-annual variability, with possibly even higher densities in open waters to the north. Yet the relatively high density of $C$. antarctica under ice and its diel vertical migration into the ice-water interface layer indicates a certain relevance of ice-water interface layer for the pteropod in winter (Fig. 6A). The following tentative rationale could explain the seasonally divergent abundance pattern with respect to the presence of sea ice: $C$. antarctica is a monophagus predator on another pteropod, Limacina helicina (Lalli and Gilmer, 1989). Limacina helicina were abundant in SUIT catches from all three sampling seasons, but were excluded from the present analysis due to their small size. Limacina helicina mainly feed on phytoplankton in summer, but little is known how they survive the winter in Antarctic ice-covered waters (Lalli and Gilmer, 1989). In the Arctic, juvenile $L$. helicina have been reported to rely on particulate organic matter originating from the sea ice in winter (Gannefors et al., 2005; Kobayashi, 1974). If that is also the case in the Southern Ocean, L. helicina is likely to concentrate under ice in winter and prefer the phytoplankton-rich open waters in summer. Its predator $C$. antarctica can be expected to follow this behavior. Clione antarctica and its prey L. helicina have a high lipid content and are thus rich in energy, making them potentially significant vectors in the energy transfer between the sea ice community and the pelagic food web in winter (Gannefors et al., 2005; Phleger et al., 1997).

A more obvious association with sea ice was evident from the amphipod Eusirus laticarpus. There was no evidence of the amphipod in the largely open surface layer in autumn, frequent 


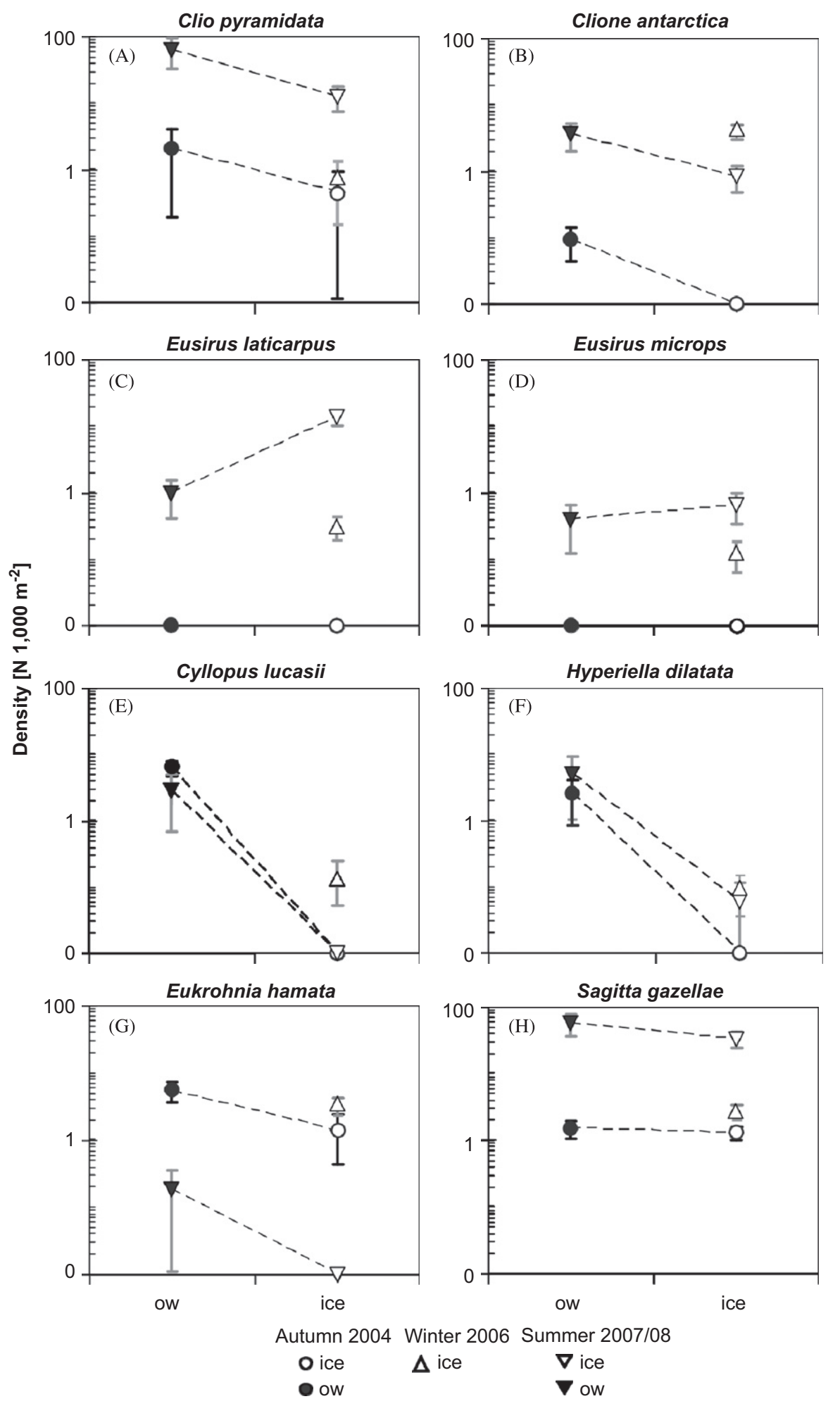

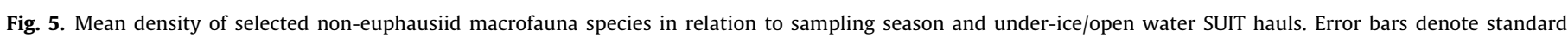
errors. Y-axes in logarithmic scale. Dashed line indicates relationship between open water and under-ice samples. Symbols as indicated in Fig. 3.

occurrence under ice in winter at moderate abundances, as well as a wide distribution and significantly higher abundances under ice than in open water in summer (Fig. 5; Table 2; Table 4). These observations suggest a pelago-sympagic life cycle of E. laticarpus that was hypothesized by Krapp et al. (2008) based on a subset of our dataset from winter. In combination with our observations from summer $2007 / 2008$, the following tentative life cycle may be proposed (Fig. 8):

- In autumn, E. laticarpus were neither caught in the ice-free, nor in the ice-covered surface layer, indicating that they do not arrive at the ice-water interface layer before early winter. 
(A)

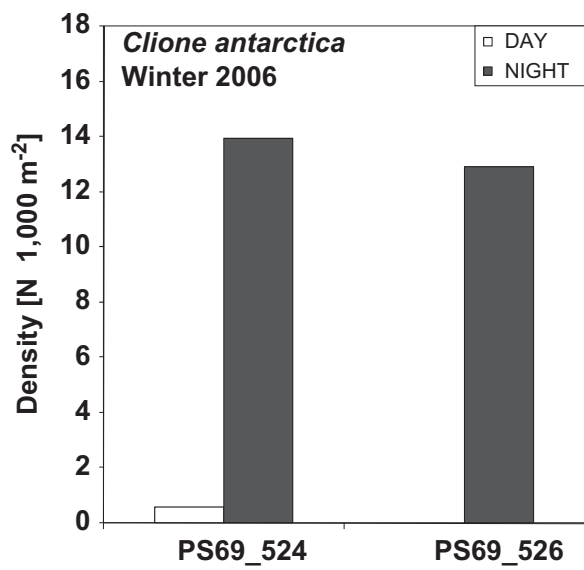

(B)

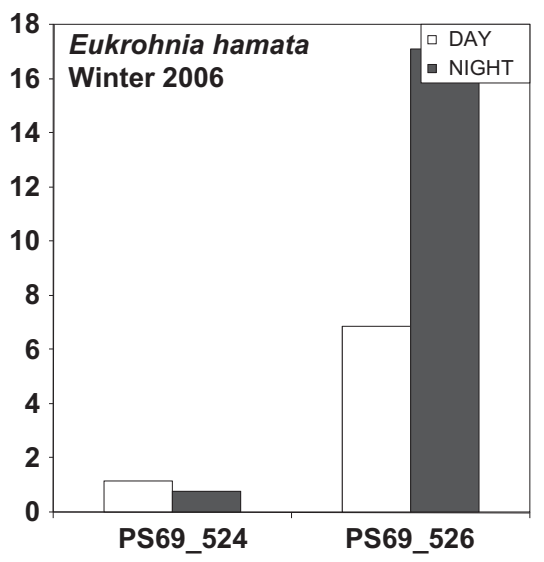

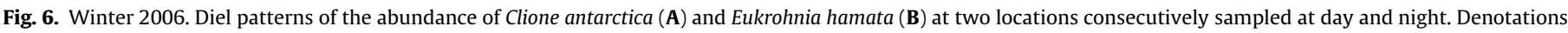
on $\mathrm{x}$-axis refer to location codes. All SUIT hauls were conducted under ice.

- In winter, they were more abundant under the older sea ice in the south and absent from this habitat in northerly areas. The results from early summer show that the distribution of E. laticarpus covers the entire sea ice area at the beginning of the melting season. This suggests that the amphipods successively occupy the ice habitat from south to north.

- In summer, the occurrence of female E. laticarpus with brood sacs under sea ice indicated that reproduction takes place at the end of the sea ice season (H.F., unpublished data). Juveniles are released into the water, and the amphipods can be assumed to return to the pelagic phase of their life cycle.

The life strategy proposed here for E. laticarpus, however, leaves many questions open. For example, investigations of the diet are urgently needed in order to assess this species' trophic interaction with sea-ice biota. It is further still unclear, how the amphipod survives the summer months. E. laticarpus was often not distinguished from the morphologically similar E. antarcticus in earlier taxonomic studies (De Broyer and Jazdzewski, 1993). A direct trophic link of E. laticarpus with sea ice biota could thus be indicated by reports of $E$. antarcticus grazing on the underside of ice floes (Hamner et al., 1989; Hopkins and Torres, 1988). Only a few potential records were reported from pelagic sampling, dating from times before the species was separated from E. antarcticus (Boysen-Ennen and Piatkowski, 1988; Hopkins and Torres, 1988; De Broyer and Jazdzewski, 1993). Thus, it is also possible that they stay in bathypelagic layers when sea ice is absent, or even switch to a benthic mode of life.

A similar pattern of presence in the ice-water interface layer was observed in E. microps, which was also suggested to be a potentially sympagic species (Krapp et al., 2008). However, its under-ice abundances were not significantly above open water values in summer (Fig. 5D), indicating either a generally more pelagic mode of life or an earlier switch to the pelagic phase in this species. In the Arctic Ocean, amphipods often dominate the sympagic macrofauna community of the ice-water interface layer (Arndt and Swadling, 2006). In the Southern Ocean, amphipods have adapted to a multitude of habitats, but sympagic amphipods have been documented only on the shelf so far (Arndt and Swadling, 2006). E. antarcticus (i.e. possibly E. laticarpus) has been reported to be preyed on by the Antarctic fish Trematomus newnesi (Vacchi and La Mesa, 1995). As foragers at the ice-water interface, E. laticarpus and E. microps could form a hitherto unnoticed trophic link from the sea ice to the pelagic species community as well as directly to the air-breathing top predators.
Species that were abundant both under ice and in the open surface layer, such as Clio pyramidata and Sagitta gazellae, may also contribute to the exchange between the sea ice system and the water column (Fig. 5A and $\mathrm{H}$ ). A high potential with respect to energy transfer from both the ice-water interface layer and the open surface layer to the water column may be expected from $S$. gazellae, which as a single species was second in wet mass only to E. superba under ice in summer $2007 / 2008$ (Table 3). This predator forages on copepods, euphausiids and other zooplankton that can be assumed to rely on surface layer and under-ice resources (Froneman and Pakhomov, 1998; Froneman et al., 1998). Through its high predation impact and fast-sinking fecal pellets, S. gazellae contributes significantly to the biological carbon pump (Giesecke et al., 2010). The high abundance of the arrow worm in the open surface layer and under sea ice indicates that the contribution of $S$. gazellae to the biological carbon pump may be even more pronounced.

Similarly, a high relevance in the trophic transfer from the icewater interface layer can be expected from ctenophores. In summer 2007/2008, ctenophores dominated the open-water community by wet mass (Fig. 2). Under sea ice, all ctenophores together accounted for the second largest wet mass after Antarctic krill, though in terms of individual species B. forskalii was third after $S$. gazellae. Ctenophores were equal in wet mass density to $S$. gazellae under the winter sea ice. The biomass contribution of ctenophores to Antarctic zooplankton is known to vary considerably between regions, seasons and years (Kaufmann et al., 2003; Lancraft et al., 1989, 1991; Voronina et al., 1994). Gut analysis and fatty acid composition of the two most abundant ctenophores found in our study, Beroe forskalii and Callianira antarctica, indicate that they feed predominantly on copepods and krill larvae (Ju et al., 2004; Nelson et al., 2000; Scolardi et al., 2006). Callianira antarctica has been observed feeding on krill larvae under sea ice (Daly and Macaulay, 1991; Hamner and Hamner, 2000; Hamner et al., 1989; Scolardi et al., 2006). Taking into account that density and biomass of ctenophores under sea ice may have been under-estimated due to disruption by ice particles in the net, our results agree with the reported feeding behavior of B. forskalii and $C$. antarctica, which indicates a seasonal feeding in the ice-water interface layer at least in C. antarctica.

The pronounced diel patterns in the occurrence of abundant species in the surface layer (Fig. 6; Fig. 7) reflected the diel vertical migration (DVM) described for a number of these species (Donnelly et al., 2006; Lancraft et al., 1989; Nordhausen, 1994). In winter 2006, these diel patterns were consistent with acoustic 

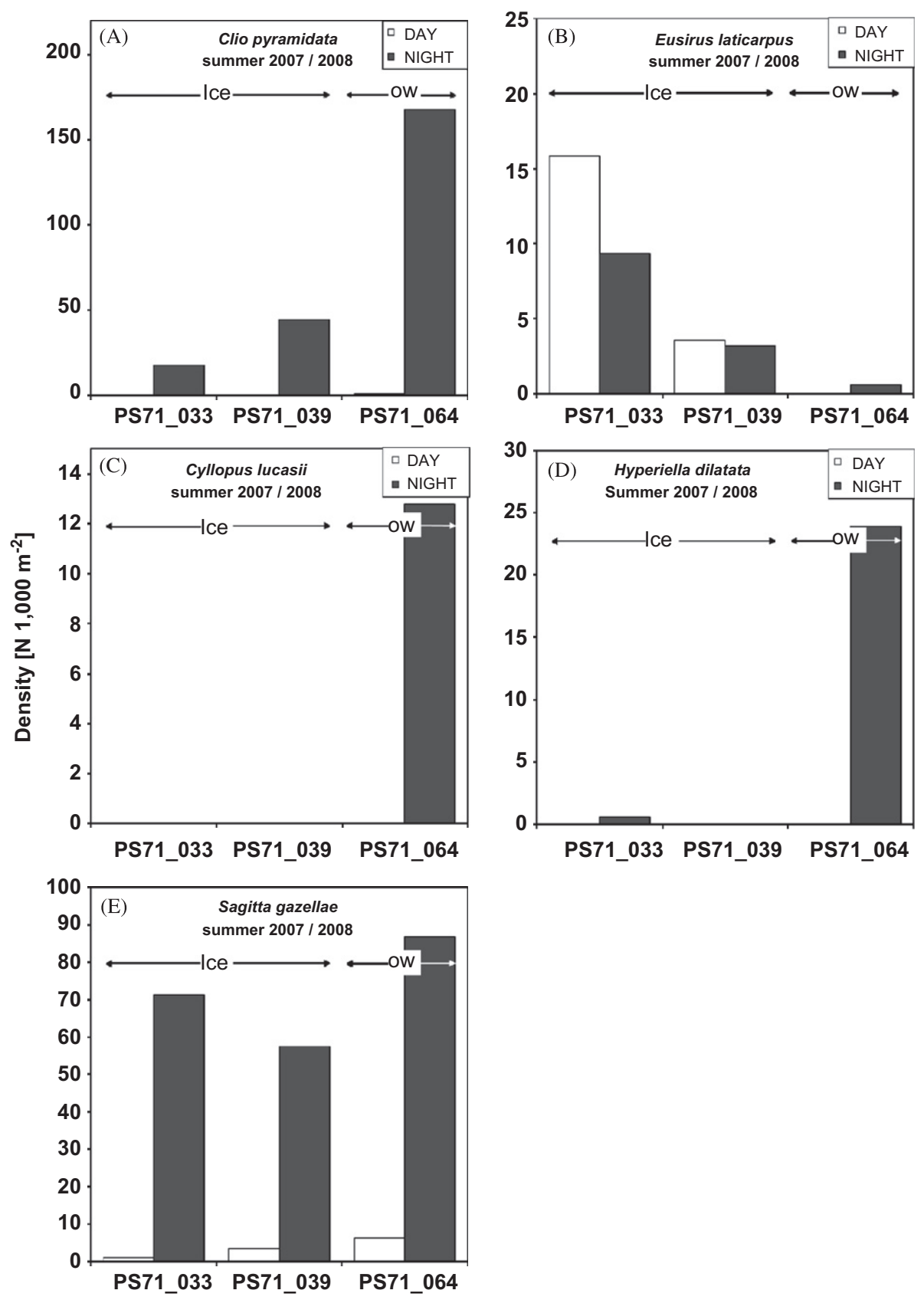

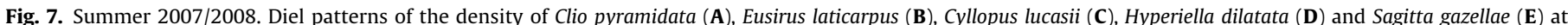

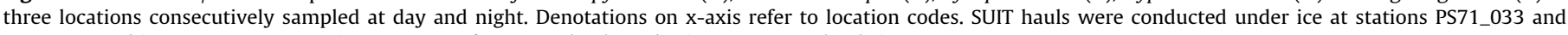
PS71_039 and in open water at station PS71_064. ice=SUIT hauls under ice; ow=SUIT hauls in open water.

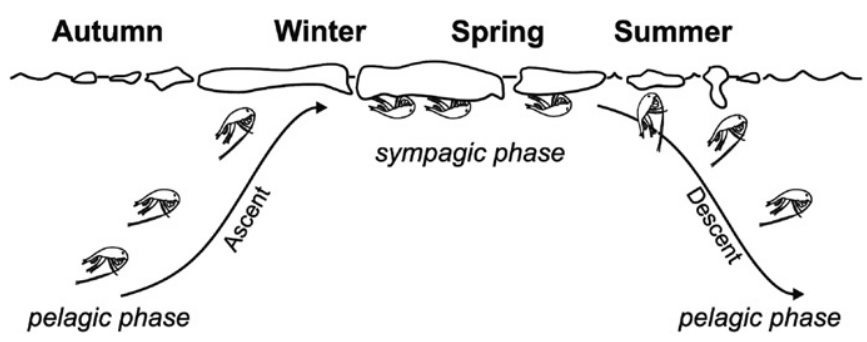

Fig. 8. Eusirus laticarpus, proposed pelago-sympagic mode of life. In early winter, the sympagic phase begins when amphipods occupy the underside of ice floes, with increasing suitability of the sea ice habitat from south to north. They stay there until the ice breaks up in summer. During the melting season, the juveniles are released, and the amphipods return to the pelagic phase of their life cycle. observations showing that the bulk of the zooplankton leaves the surface layer during day (Cisewski et al., 2010). Cisewski et al. (2010) suggested that DVM ceases completely during summer. Conversely, abundances of Antarctic krill and E. laticarpus were similar at day and at night in summer $2007 / 2008$, indicating that visual net avoidance was not a significant factor (Fig. 7B; Flores et al., 2009). The pronounced diel patterns in the surface layer density of $C$. pyramidata, $C$. lucasii, $H$. dilatata and $S$. gazellae may suggest that DVM persists in these species also in summer. Due to the $20 \mathrm{~m}$ minimum sampling depth of the ADCP used, DVM between the surface layer and up to $50 \mathrm{~m}$ depth could not be detected as such by Cisewski et al. (2010) in summer (Fig. 7 A; C-E).

Because most zooplankton species performing DVM are distributed closer to the surface at night and in deeper layers at day, 
DVM may have introduced a minor negative bias to abundance and biomass estimates of our study due to the 11 daytime hauls included in our analysis. DVM is often considered as the consequence of diel changes in the balance of benefits (e.g. better food availability) and risks (e.g. visual predators) to stay in a certain depth layer and has a cost, the energy invested in vertical movement (De Robertis, 2002; Gabriel and Thomas, 1988). In that sense, the diel patterns observed in the present study indicate that both the open surface and the ice-water interface layer were an attractive environment for Antarctic macrofauna, supplying sufficient benefits to compensate for the cost of vertical migration in spite of their exposure to ice, atmosphere, and visual predators.

An accurate assessment of the importance of macrofauna dwelling under ice and in the open surface layer for the pelagic food web, however, is beyond the possibilities of this baseline investigation. Complete answers to the underlying ecological processes are only possible with a better knowledge on the biology, diet composition and spatiotemporal distribution of relevant species in the water column, both in the open surface layer and under ice, including mesozooplankton and nekton. Squid and fish for example mostly feed on copepods and other zooplankton, potentially allocating much of the energy from the open surface and the ice-water interface layer into the mesopelagic realm, and ultimately to the air-breathing top predators (Hopkins et al., 1993). Our study records occasional catches of three species of postlarval fish (Aethotaxis mitopteryx, Electrona antarctica, Notolepis sp.) and one species of adult squid (Kondakovia longimana) in the LAKRIS grid, as well as an additional species of squid (Slosarczykovia circumantarctica) at a station excluded from this study in the marginal ice zone north of $60^{\circ} \mathrm{S}$ in winter 2006 (van Franeker et al., 2009). The true abundances of surface-dwelling nekton may have been higher due to net avoidance. Yet, the evidence of fish and squid in the 0-2 m stratum supports the high importance of the open surface and ice-water interface layer as a foraging ground for pelagic nekton and air-breathing top predators suggested by Ainley et al. (1986).

\subsection{Conclusions}

Plasticity in terms of taxonomic composition, mode of life and size characterized the species community of the $0-2 \mathrm{~m}$ surface layer in the Lazarev Sea. Rather than being a depleted border region of the pelagic realm, the surface layer attracts most species found over much greater depth ranges. Through (seasonal) association with sea ice in some species and the avoidance of the ice by others, the community composition significantly responds to the occurrence of sea ice. Besides the often dominant Antarctic krill, a range of other abundant species may be important in the energy transfer from sea ice biota into the pelagic food web, including ctenophores, pteropods, amphipods and chaetognaths. New evidence for an ice-associated mode of life in at least one oceanic amphipod species illustrates that the ice-water interface layer can be important for the biodiversity of the Southern Ocean as a temporary habitat for a variety of unique species.

The results of the present study provide new quantitative evidence that the ice-water interface layer is an important functional node in the ecosystem of the Antarctic sea ice zone almost year-round. However, the role of important components of the sea ice food web, such as ctenophores, pteropods and fish, is barely understood. A concise investigation of the trophic interactions between the sea ice system, near-surface plankton and nekton and the pelagic food web including deeper layers is the next logical step to gain a more accurate picture of the ecological importance of the ice-water interface and the open surface layer in the Southern Ocean.

\section{Acknowledgments}

This study was made possible by extraordinary support of the crews of the Polarstern expeditions ANT XXI-4, ANT XXIII-6 and ANT XXIV-2, Captain Uwe Pahl, Captain Stefan Schwarze and the Alfred Wegener Institute for Polar and Marine Research (AWI). The following people contributed substantially to the successful completion of our research: A. Meijboom, M. van Dorssen, R. Fijn, B. Fey, D. Martynova, H. Verdaat, I. Nunez-Riboni, U. Piatkowski and M. Scheidat. This Antarctic research by IMARES WageningenUR is commissioned by the Netherlands Ministry of Agriculture, Nature and Food Quality (LNV) under its Statutory Research Task Nature \& Environment WOT-04-003-002, and project no KB-01012-002. The Netherlands AntArctic Program (NAAP), managed by the Netherlands Organization for Scientific Research (NWO) funded this research under Project nr ALW 851.20.011. A.VdP. is supported by Belgian Science Policy (PELAGANT project and the PADI project no. SD/BA/851). H.L.'s participation was supported by a travel grant from the Royal Society.

\section{References}

Ainley, D.G., Fraser, W.R., Smith, W.O.J., Hopkins, T.L., Torres, J.J., 1991. The structure of upper level pelagic food webs in the Antarctic: effect of phytoplankton distribution. J Mar Sys 2, 111-122.

Ainley, D.G., Fraser, W.R., Sullivan, C.W., Torres, J.J., Hopkins, T.L., Smith, W.O. 1986. Antarctic mesopelagic micronekton: Evidence from seabirds that pack ice affects community structure. Science 232, 847-849.

Ainley, D.G., Tynan, C.T., Stirling, I., 2003. Sea ice: a critical habitat for polar marine mammals and birds. In: Thomas, D.N., Dieckmann, G. (Eds.), Sea ice - an introduction to its physics, chemistry, biology and geology. Blackwell Science, Oxford, pp. 240-266.

Arndt, C.E., Swadling, K.M., 2006. Crustacea in Arctic and Antarctic sea ice: distribution, diet and life history strategies. Adv Mar Biol 51, 197-315.

Arrigo, K.R., Thomas, D.N., 2004. Large scale importance of sea ice biology in the Southern Ocean. Ant Sci 16, 471-486.

Arrigo, K.R., Worthen, D.L., Lizotte, M.P., Dixon, P., Dieckmann, G., 1997. Primary production in Antarctic sea ice. Science 276, 394-397.

Bluhm, B.A., Gradinger, R., Piraino, S., 2007. First record of sympagic hydroids (Hydrozoa, Cnidaria) in Arctic coastal fast ice. Polar Biol 30, 1557-1563.

Boysen-Ennen, E., Piatkowski, U., 1988. Meso- and macrozooplankton communities in the Weddell Sea, Antarctica. Polar Biol 9, 17-35.

Bray, J.R., Curtis, J.T., 1957. An ordination of upland forest communities of southern Wisconsin. Ecol Monogr 27, 325-349.

Brierley, A.S., Fernandes, P.G., Brandon, M.A., Armstromg, F., Millard, N.W. McPhail, S.D., Stevenson, P., Pebody, M., Perrett, J., Squires, M., Bone, D.G., Griffiths, G., 2002. Antarctic krill under sea ice: Elevated abundance in a narrow band just south of ice edge. Science 295, 1890-1892.

Brierley, A.S., Thomas, D.N., 2002. Ecology of Southern Ocean pack ice. Adv Mar Biol 43, 171-276.

Cisewski, B., Strass, V.H., Rhein, M., Krägefsky, S., 2010. Seasonal variation of diel vertical migration of zooplankton from ADCP backscatter time series data in the Lazarev Sea, Antarctica. Deep-Sea Res. I 57, 78-94.

Daly, K.L., 2004. Overwintering growth and development of larval Euphausia superba: an interannual comparison under varying environmental conditions west of the Antarctic Peninsula. Deep-Sea Res. II 51, 2139-2168.

Daly, K.L., Macaulay, M., 1991. The influence of physical and biological mesoscale dynamics on the seasonal distribution and behavior of Euphausia superba in the Antarctic marginal ice zone. Mar Ecol Prog Ser 79, 37-66.

De Broyer, C., Jazdzewski, K., 1993. Contribution to the marine biodiversity inventory: a checklist of the Amphipoda (Crustacea) of the Southern Ocean Studiedocumenten van het K.B.I.N., 1-154.

De Robertis, A., 2002. Size-dependent visual predation risk and the timing of vertical migration: an optimization model. Limnol Oceanogr 47, 925-933.

Donnelly, J.J., Sutton, T.T., Torres, J.J., 2006. Distribution and abundance of micronekton and macrozooplankton in the NW Weddell Sea: relation to a spring ice-edge bloom. Polar Biol 29, 280-293.

Eicken, H., 1992. The role of sea ice in structuring Antarctic ecosystems. Polar Biol $12,3-13$.

Fisher, E.C., Kaufmann, R.S., Smith, K.L.J., 2004. Variability of epipelagic macrozooplankton/micronekton community structure in the NW Weddell Sea, Antarctica (1995-1996). Mar Biol 144, 345-360.

Flores, H., Van Franeker, J.A., Siegel, V., Haraldsson, M., Strass, V., Meesters, H.W.G. Bathmann, U., Wolff, W.J., 2009. Antarctic krill species (Crustacea: Euphausiidae) 
under sea ice and in the open surface layer, in: Flores, H. (Ed.), Frozen Desert Alive. PhD thesis. University of Groningen, Groningen, pp. 155-180. 〈http:// dissertations.ub.rug.nl/faculties/science/2009/h.flores/ $>$.

Froneman, P.W., Pakhomov, E.A., 1998. Trophic importance of the chaetognaths Eukrohnia hamata and Sagitta gazellae in the pelagic system of the Prince Edward Islands (Southern Ocean). Polar Biol 19, 242-249.

Froneman, P.W., Pakhomov, E.A., Perissinotto, R., McQuaid, C.D., 2000. Zooplankton structure and grazing in the Atlantic sector of the Southern Ocean in late austral summer 1993 - Part 2. Biochemical zonation. Deep-Sea Research I 47, 1687-1702.

Froneman, P.W., Pakhomov, E.A., Perissinotto, R., Meaton, V., 1998. Feeding and predation impact of two chaetognath species, Eukrohnia hamata and Sagitta gazellae, in the vicinity of Marion Island (southern ocean). Mar Biol 131, 95-101.

Gabriel, W., Thomas, B., 1988. Vertical migration of zooplankton as an evolutionarily stable strategy. American Naturalist 132, 199-216.

Gannefors, C., Boer, M., Kattner, G., Graeve, M., Eiane, K., Gulliksen, B., Hop, H., Falk-Petersen, S., 2005. The Arctic sea butterfly Limacina helicina: lipids and life strategy. Mar Biol 147, 169-177.

Giesecke, R. Gonzalez, H.E., Bathmann, U., 2010. The role of the chaetognath Sagitta gazellae in the vertical carbon flux of the Southern Ocean. Polar Biol 33 293-304.

Gulliksen, G., Lonne, O.J., 1991. Sea ice macrofauna in the Antarctic and the Arctic J Mar Sys 2, 53-61.

Hamner, W., 1982. Procedures for in situ observations of krill schools in the Southern Ocean. Antarct. J. U.S. 8, 165.

Hamner, W., Hamner, P., 2000. Behavior of Antarctic krill (Euphausia superba): schooling, foraging, and antipredatory behavior. Can J Fish Aq Sci 57 (Suppl. 3) $192-202$.

Hamner, W., Hamner, P., Obst, B., 1989. Field observations on the ontogeny of schooling of Euphausia superba furciliae and its relationship to ice in Antarctic waters. Limnol Oceanogr 34, 451-456.

Hardy, A., Gunther, E., 1935. The plankton of the South Georgia whaling grounds and adjacent waters, 1926-1927. Discov Rep 1.

Hopkins, T.L., Ainley, D.G., Torres, J.J., Lancraft, T.M., 1993. Trophic structure in open waters of the marginal ice zone in the Scotia-Weddell confluence region during spring (1983). Polar Biol 13, 389-397.

Hopkins, T.L., Torres, J.J., 1988. The zooplankton community in the vicinity of the ice edge, western Weddell Sea, March 1986. Polar Biol 9, 79-87.

Hunt, B.P.V., Pakhomov, E.A., Siegel, V., Strass, V., Cisewski, B., Bathmann, U. The seasonal cycle of the Lazarev Sea macrozooplankton community and a potential shift to top-down trophic control in winter. Deep-Sea Res. II, in press [doi:10.1016/j.dsr2.2010.11.016]

Hunt, B.P.V., Pakhomov, E.A., Trotsenko, B.G., 2007. The macrozooplankton of the Cosmonaut Sea, east Antarctica ( $30^{\circ} \mathrm{E}$ to $\left.60^{\circ} \mathrm{E}\right), 1987-1990$. Deep-Sea Res. I 54 1042-1069.

Ju, S.-J., Scolardi, K., Daly, K.L., Harvey, H.R., 2004. Understanding the trophic role of the Antarctic ctenophore, Callianira antarctica, using lipid biomarkers. Polar Biol 27, 782-792.

Kaufmann, R., Smith, K.J., Baldwin, R., Glatts, R., Robinson, B., Reisenbichler, K. 1995. Effects of seasonal pack ice on the distribution of macrozooplankton and micronecton in the northwestern Weddell Sea. Mar Biol 124, 387-397.

Kaufmann, R.S., Fisher, E.C., Gill, W.H., King, A.L., Laubacher, M., Sullivan, B., 2003. Temporal patterns in the distribution, biomass and community structure of macrozooplankton and micronekton within Port Foster, Deception Island, Antarctica. Deep-Sea Res. II 50, 1765-1785.

Kiko, R., Kramer, M., Spindler, M., Wägele, H., 2008a. Tergipes antarcticus (Gastropoda, Nudibranchia): distribution, life cycle, morphology, anatomy and adaptation of the first mollusc known to live in Antarctic sea ice. Polar Biol 31, 1383-1395.

Kiko, R., Michels, J., Mizdalski, E., Schnack-Schiel, S.B., Werner, I., 2008b. Living conditions, abundance and composition of the metazoan fauna in surface and sub-ice layers in pack ice of the western Weddell Sea during late spring. DeepSea Res. II 55, 1000-1014.

Kils, U., 1979. Swimming speed and escape capacity of Antarctic krill, Euphausia superba. Meeresforsch 27, 264-266.

Kobayashi, H.A., 1974. Growth cycle and related vertical distribution of thecosomatous pteropod Spiratells (Limacina) helicina in central Arctic ocean. Mar Biol 26, 295-301.

Krapp, R.H., Berge, J., Flores, H., Gulliksen, B., Werner, I., 2008. Sympagic occurrence of Eusirid and Lysianassoid amphipods under Antarctic pack ice. Deep-Sea Res. II 55, 1015-1023.

Kruskal, J.B., 1964. Nonmetric Multidimensional Scaling - a numerical method. Psychometrika 29, 115-129.

Lalli, C.M., Gilmer, R.W., 1989. Pelagic snails. The biology of holoplanktonic gastropod mollusks. Stanford University Press, Stanford, California.
Lancraft, T.M., Hopkins, T.L., Torres, J.J., Donnelly, J., 1991. Oceanic micronektonic / macrozooplanktonic community structure and feeding in ice-covered Antarctic waters during the winter (AMERIEZ 1988). Polar Biol 11, 157-167.

Lancraft, T.M., Torres, J.J., Hopkins, T.L., 1989. Micronekton and macrozooplankton in the open waters near Antarctic ice edge zones (AMERIEZ 1983 and 1986). Polar Biol 9, 225-233.

Lizotte, M., 2001. The contribution of sea ice algae to Antarctic marine primary production. Americ Zool 41, 57-73.

Marschall, H.P., 1988. The overwintering strategy of Antarctic krill under the packice of the Weddell Sea. Polar Biol 9, 129-135.

McMinn, A., Pankowskii, A., Ashworth, C., Bhagooli, R., Ralph, P., Ryan, K., 2010. In situ net primary productivity and photosynthesis of Antarctic sea ice algal, phytoplankton and benthic algal communities. Mar Biol 157, 1345-1356.

Nelson, M., Phleger, C., Mooney, B., Nichols, P., 2000. Lipids of gelatinous Antarctic zooplankton: Cnidaria and Ctenophora. Lipids 35, 551-559.

Nemoto, T., 1983. Net sampling and abundance estimates of euphausiids. Biological Oceanography 2, 211-226.

Nordhausen, W., 1994. Winter abundance and distribution of Euphausia superba E. crystallorophias, and Thysanoessa macrura in Gerlache Strait and Crystal Sound, Antarctica. Mar Ecol Prog Ser 109, 131-142.

Oksanen, J., Kindt, R., Legendre, P., O'Hara, R.B., Simpson, G.L., Stevens, M.H.H., 2008. Vegan: Community ecology package version 1.8.3. R Foundation for Statistical Computing.

Pakhomov, E.A., Froneman, P.W., 2000. Composition and spatial variability of macroplankton and micronekton within the Antarctic Polar Frontal Zone of the Indian Ocean during austral autumn 1997. Polar Biol 23, 410-419.

Phleger, C.F., Nichols, P.D., Virtue, P., 1997. Lipids and buoyancy in southern ocean pteropods. Lipids 32, 1093-1100.

Piatkowski, U., 1989. Macroplankton communities in Antarctic surface waters spatial changes related to hydrography. Mar Ecol Prog Ser 55, 251-259.

R-Development-Core-Team, 2008. R: A language and environment for statistical computing. R Foundation for Statistical Computing, Vienna.

Ross, R.M., Quetin, L.B., Haberman, K.L., 1998. Interannual and seasonal variability in short-term grazing impact of Euphausia superba in nearshore and offshore waters west of the Antarctic Peninsula. J Mar Sys 17, 261-273.

Schnack-Schiel, S.B., 2003. The macrobiology of sea ice. In: Thomas, D.N., Dieckmann, G. (Eds.), Sea ice - an introduction to its physics, chemistry, biology and geology.. Blackwell Science, Oxford, pp. 211-239.

Schnack-Schiel, S.B., Haas, C., Michels, J., Mizdalski, E., Schunemann, H., Steffens, M., Thomas, D.N., 2008. Copepods in sea ice of the western Weddell Sea during austral spring 2004. Deep-Sea Res. II 55, 1056-1067.

Scolardi, K., Daly, K., Pakhomov, E., Torres, J., 2006. Feeding ecology and metabolism of the Antarctic cydippid ctenophore Callianira antarctica. Mar Ecol Prog Ser 317, 111-126.

Siegel, V., Bergström, B., Stromberg, J.O., Schalk, P.H., 1990. Distribution, size frequencies and maturity stages of Krill, Euphausia superba, in relation to sea ice in the northern Weddell Sea. Polar Biol 10, 549-557.

Siegel, V., Skibowski, A., Harm, U., 1992. Community structure of the epipelagic zooplankton community under the sea-ice of the northern Weddell Sea. Polar Biol 12, 15-24.

Stretch, J.J., Hamner, P.P., Hamner, W.M., Michel, W.C., Cook, J., Sullivan, C.W., 1988. Foraging behavior of Antarctic krill Euphausia superba on sea ice microalgae. Mar Ecol Prog Ser 44, 131-139.

Tanimura, A., Hattori, H., Miyamoto, Y., Hoshiai, T., Fukuchi, M., 2008. Diel changes in vertical distribution of Oithona similis (Cyclopoida) and Oncaea curvata (Poecilostomatoida) under sea ice in mid-summer near Syowa Station, Antarctica. Polar Biol 31, 561-567

Vacchi, M., La Mesa, M., 1995. The diet of the Antarctic fish Trematomus newnesi Boulenger, 1902 (Nototheniidae) from Terra Nova Bay, Ross Sea. Ant Sci 7 37-38.

van Franeker, J.A., Bathmann, U.V., Mathot, S., 1997. Carbon fluxes to Antarctic top predators. Deep-Sea Res. II 44, 435-455.

van Franeker, J.A., Flores, H., Van Dorssen, M., 2009. The Surface and Under Ice Trawl (SUIT). In: Flores, H. (Ed.), Frozen Desert Alive.. University of Groningen, Groningen, pp. 181-188 pp.

Voronina, N.M., Kosobokova, K.N., Pakhomov, E.A., 1994. Composition and biomass of summer metazoan plankton in the 0-200 m layer of the Atlantic sector of the Antarctic. Polar Biol 14, 91-95.

Watanabe, Y., Bornemann, H., Liebsch, N., Plotz, J., Sato, K., Naito, Y., Miyazaki, N., 2006. Seal-mounted cameras detect invertebrate fauna on the underside of an Antarctic ice shelf. Mar Ecol Prog Ser 309, 297-300.

Zhou, M., Nordhausen, W., Huntley, M., 1994. ADCP Measurements of the distribution and abundance of euphausiids near the Antarctic Peninsula in winter. Deep-Sea Res. I 41, 1425-1445. 